\title{
Massive star cluster formation and evolution in tidal dwarf galaxies ${ }^{\star}$
}

Jérémy Fensch ${ }^{1,2}$, Pierre-Alain Duc ${ }^{3,2}$, Médéric Boquien $^{4}$, Debra M. Elmegreen ${ }^{5}$, Bruce G. Elmegreen ${ }^{6}$, Frédéric Bournaud $^{2}$, Elias Brinks ${ }^{7}$, Richard de Grijs ${ }^{8,9,10}$, Federico Lelli ${ }^{1}$, Florent Renaud ${ }^{11}$, and Peter M. Weilbacher ${ }^{12}$

${ }^{1}$ European Southern Observatory, Karl-Schwarzschild-Str. 2, 85748 Garching, Germany e-mail: jfensch@eso.org, jeremy.fensch@gmail.com

2 AIM, CEA, CNRS, Université Paris-Saclay, Université Paris Diderot, Sorbonne Paris Cité, 91191 Gif-sur-Yvette, France

3 Université de Strasbourg, CNRS, Observatoire astronomique de Strasbourg, UMR 7550, 67000 Strasbourg, France

${ }^{4}$ Centro de Astronomía (CITEVA), Universidad de Antofagasta, Avenida Angamos 601, Antofagasta, Chile

5 Vassar College, Dept. of Physics and Astronomy, Poughkeepsie, NY, USA

6 IBM Research Division, T.J. Watson Research Center, 1101 Kitchawan Road, Yorktown Heights, NY 10598, USA

7 Centre for Astrophysics Research, School of Physics, Astronomy and Mathematics, University of Hertfordshire, Hatfield, Herts AL10 9AB, UK

${ }^{8}$ Department of Physics and Astronomy, Macquarie University, Balaclava Road, Sydney, NSW 2109, Australia

9 Research Centre for Astronomy, Astrophysics and Astrophotonics, Macquarie University, Balaclava Road, Sydney, NSW 2109, Australia

10 International Space Science Institute-Beijing, 1 Nanertiao, Zhongguancun, Hai Dian District, Beijing 100190, PR China

11 Lund Observatory, Department of Astronomy and Theoretical Physics, Box 43, 22100 Lund, Sweden

12 Leibniz-Institut für Astrophysik (AIP), An der Sternwarte 16, 14482 Potsdam, Germany

Received 8 October 2018 / Accepted 6 June 2019

\begin{abstract}
Context. The formation of globular clusters remains an open debate. Dwarf starburst galaxies are efficient at forming young massive clusters with similar masses as globular clusters and may hold the key to understanding their formation.

Aims. We study star cluster formation in a tidal debris, including the vicinity of three tidal dwarf galaxies, in a massive gas-dominated collisional ring around NGC 5291. These dwarfs have physical parameters that differ significantly from local starbursting dwarfs. They are gas rich, highly turbulent, their gas metallicity is already enriched up to half solar values, and they are expected to be free of dark matter. The aim is to study massive star cluster formation in this as yet unexplored type of environment.

Methods. We used imaging from the Hubble Space Telescope using broadband filters that cover the wavelength range from the nearultraviolet to the near-infrared. We determined the masses and ages of the cluster candidates by using the spectral energy distributionfitting code CIGALE. We considered age-extinction degeneracy effects on the estimation of the physical parameters.

Results. We find that the tidal dwarf galaxies in the ring of NGC 5291 are forming star clusters with an average efficiency of $~ 40 \%$, which is similar to blue compact dwarf galaxies. We also find massive star clusters for which the photometry suggests that they were formed at the very birth of the tidal dwarf galaxies. These clusters have survived for several hundred million years. Therefore our study shows that extended tidal dwarf galaxies and compact clusters may be formed simultaneously. In the specific case observed here, the young star clusters are not massive enough to survive for a Hubble time. However, it may be speculated that similar objects at higher redshift, with a higher star formation rate, might form some of the long-lived globular clusters.
\end{abstract}

Key words. galaxies: dwarf - galaxies: irregular - galaxies: star clusters: general - galaxies: stellar content galaxies: star formation - galaxies: interactions

\section{Introduction}

Globular clusters (GCs) are among the oldest stellar structures in the Universe. Their formation redshift is estimated to be around $z=2-6$ based on their stellar population, but their formation channels are still debated (see reviews by Forbes et al. 2018; Renaud 2018). In particular, the formation environment must be able to host very dense and massive gas clouds to allow the formation of these bound stellar clusters. Based on theoretical

\footnotetext{
* Photometry data are only available at the CDS via anonymous ftp to cdsarc.u-strasbg. fr (130.79.128.5) or via http://cdsarc.
} u-strasbg.fr/viz-bin/qcat?J/A+A/628/A60 grounds, it has for instance been proposed that galaxy mergers at high redshift could be an important formation channel of current metal-rich GC populations (Ashman \& Zepf 1992; Li \& Gnedin 2014; Kim et al. 2018). Giant gas clumps in high-redshift gasdominated galaxies could also host a favorable environment for GC formation (Shapiro et al. 2010; Kruijssen 2015). The metalpoor part of the GC populations is proposed to be formed in the high-redshift highly turbulent gas-rich dwarf galaxies, such as the little blue dots seen at redshifts $0.5-4$ in the Hubble Frontier Fields (Elmegreen \& Elmegreen 2017), which would be accreted, with their GC populations, onto more massive galaxies (Côté et al. 1998; Elmegreen et al. 2012a; Tonini 2013; 
Renaud et al. 2017). Unfortunately, current instrumentation cannot probe the physical conditions of the GC birth environment at high redshifts, except for exceptional cases of strong gravitational lenses (Vanzella et al. 2017a,b; Bouwens et al. 2017). Observational studies of star cluster formation have thus so far mainly focused on favorable environments for massive star cluster formation in the Local Universe.

Local dwarf galaxies are particularly interesting for the problem of GC formation. In starbursting dwarfs, young massive star clusters (YMCs) with masses above $10^{5} M_{\odot}$ and radii around $3 \mathrm{pc}$ are typically found, which is in the mass and size range of GCs (see, e.g., de Grijs et al. 2013; Hunter et al. 2016, and references therein). Furthermore, in local starbursting dwarf galaxies, such as the blue compact dwarf galaxies (BCDGs), up to $50 \%$ of their current star formation rate (SFR) can occur in YMCs (see, e.g., Adamo et al. 2011). Finally, old evolved dwarf galaxies typically have a very large number of old GCs per unit luminosity, also called specific frequency $\left(S_{N}\right)$ defined as the number of GCs per unit -15 absolute magnitude in the $V$-band $\left(M_{V}\right): \mathrm{S}_{\mathrm{N}}=\mathrm{N}_{\mathrm{GC}} \times 10^{0.4\left(M_{V}+15\right)}$. It is much larger for old evolved dwarfs than for late-type galaxies and similar to massive early-type galaxies (see, e.g., Lotz et al. 2004; Peng et al. 2008; Georgiev et al. 2010). Although the formation conditions of present-day YMCs and old GCs are most certainly significantly different, these observations suggest that dwarf galaxies provide a favorable environment for both the formation and the survival of massive star clusters.

To extend the parameter space of dwarf galaxy environments, we present a study of massive star cluster formation and survival in dwarf galaxies that differ significantly from typical starbursting galaxies: tidal dwarf galaxies (TDGs). These galaxies are formed from gas and stars originating from the outskirts of a massive galaxy after a galaxy-galaxy interaction (see review by Duc et al. 1999). Because of this particular mode of formation, they are typically young and gas-dominated and are expected to be free of dark matter (DM). Most importantly, their gas content is pre-enriched in metals and may already have a metallicity of one-third to half solar. Thus, they deviate from the luminosity-metallicity diagram and have a significantly higher metallicity than starbursting dwarfs for a similar luminosity (see, e.g., Weilbacher et al. 2003). Previous studies of the formation of tidal tails have shown that star clusters and TDGs may be able to form together in some cases (Knierman et al. 2003; Mullan et al. 2011). Possible examples of tidal dwarfs at redshifts of $0.5-1$ were presented by Elmegreen et al. (2007a). It should be noted that the merger frequency was much higher at high redshift so that their contribution to the GC and TDG formation may have been larger. However, mergers at high redshift seem to be much less efficient at triggering an enhancement of star formation (Rodighiero et al. 2011; Perret et al. 2014; Lofthouse et al. 2017), probably because of their high gas fraction (Fensch et al. 2017). The formation of GCs and TDG in high-redshift galaxies still needs to be investigated.

The system studied in this paper is composed of young galaxies formed in collisional rather than in tidal debris. Even though they are thus not formally of tidal origin, they are also found in the halo of a more massive galaxy and share the same physical properties as bona fide TDGs. We therefore use the term TDGs for these galaxies as well. They are located in a huge HI ring $\left(\mathrm{M}_{\mathrm{HI}}>10^{11} M_{\odot}\right.$, Duc \& Mirabel 1998) that was expelled from the massive galaxy NGC 5291 (NED distance: 63.5 Mpc assuming the following cosmological parameters: $h=$ $73, \Omega_{\mathrm{m}}=0.27, \Omega_{\Lambda}=0.73$, distance modulus of $34.0 \mathrm{mag}$ ), most probably after an encounter with a bullet galaxy around $360 \mathrm{Myr}$ ago (Bournaud et al. 2007). This ring hosts four gravitationally bound objects with masses as high as $2 \times 10^{9} M_{\odot}$ (Lelli et al. 2015), which is in the range of dwarf galaxies. These TDGs have a gas to stellar mass fraction of $\sim 50 \%$ (Bournaud et al. 2007; Lelli et al. 2015) and their spectral energy distribution (SED) is consistent with no stellar population older than $1 \mathrm{Gyr}$ (Boquien et al. 2009). Their material has been pre-enriched inside the host galaxy: they typically show half-solar metallicity (Duc \& Mirabel 1998; Fensch et al. 2016).

This unique system has an extensive wavelength coverage: $21 \mathrm{~cm}$ HI line observations with the Very Large Array (VLA; Bournaud et al. 2007), molecular gas (Braine et al. 2001; Lelli et al., in prep.), far-infrared with Herschel (Boquien et al., in prep.), mid-infrared (IR) with Spitzer (Boquien et al. 2007), $\mathrm{H} \alpha$ with Fabry-Perot interferometry on the ESO $3.2 \mathrm{~m}$ (Bournaud et al. 2004) and optical Integral Field Unit with the Multi Unit Spectroscopic Explorer (MUSE; Fensch et al. 2016) and farand near-ultraviolet (UV) with the Galaxy Evolution Explorer (GALEX; Boquien et al. 2007). Radio and optical spectroscopy have shown the kinematical decoupling of the TDGs from the ring and their complex internal dynamics. MUSE has probed the variation in ionization processes throughout the most massive TDG of this system. However, none of the previously used instruments had the spatial resolution to investigate the TDG substructures and star cluster population.

In this paper we present optical and near-IR imaging data from the Hubble Space Telescope (HST) that cover three of these TDGs. The pixel size in the optical is $0.04^{\prime \prime}$, which corresponds to $12 \mathrm{pc}$ at the distance of NGC 5291 and is small enough to allow us to distinguish the expected YMCs that formed inside the dwarfs. We obtained broadband imaging covering a wavelength range from the near-UV to the near-IR. This allows us to derive the mass and age distributions of these TDG star cluster populations and study their formation and survival up to several hundred megayears in this particular environment.

We present the data acquisition and reduction in Sect. 2. The star cluster selection and photometry measurements are presented in Sect. 3. The derivation of their physical parameters (masses and ages) is described in Sect. 4. We discuss the cluster formation efficiency and cluster evolution in Sect. 5 and conclude the paper in Sect. 6.

\section{Observation and data reduction}

The collisional ring around NGC 5291 was observed with the WFC3 instrument on board the HST (Project ID 14727, PI: Duc). The location of the field of view and the collisional ring are shown in Fig. 1. We obtained photometry in the F336W, F475W, F606W, F814W, and F160W bands. As we discuss in Sect. 4, this set of filters was chosen for its ability to separate color effects from metallicity, age, and extinction in young star clusters (Anders et al. 2004). The respective exposure times are given in Table 1. We used the product of the regular MultiDrizzle reduction pipeline (Koekemoer 2002). The pixel size is $0.04^{\prime \prime}$ for F336W, F475W, F606W, and F814W. The pixel size is $0.12^{\prime \prime}$ for F160W. At the distance of NGC 5291, this corresponds to $12 \mathrm{pc}$ and $36 \mathrm{pc}$, respectively.

Only fields 1 and 4 were observed in the F336W and F160W bands. The field of view of the F160W data is slightly different and is shown in the right part of Fig. 1. The massive galaxy NGC 5291 and its companion (the Seashell galaxy, Duc et al. 1999 ) is visible in the top part of field 3, the TDG $\mathrm{N}$ in field 1 , and the TDGs S and SW in field 4. 


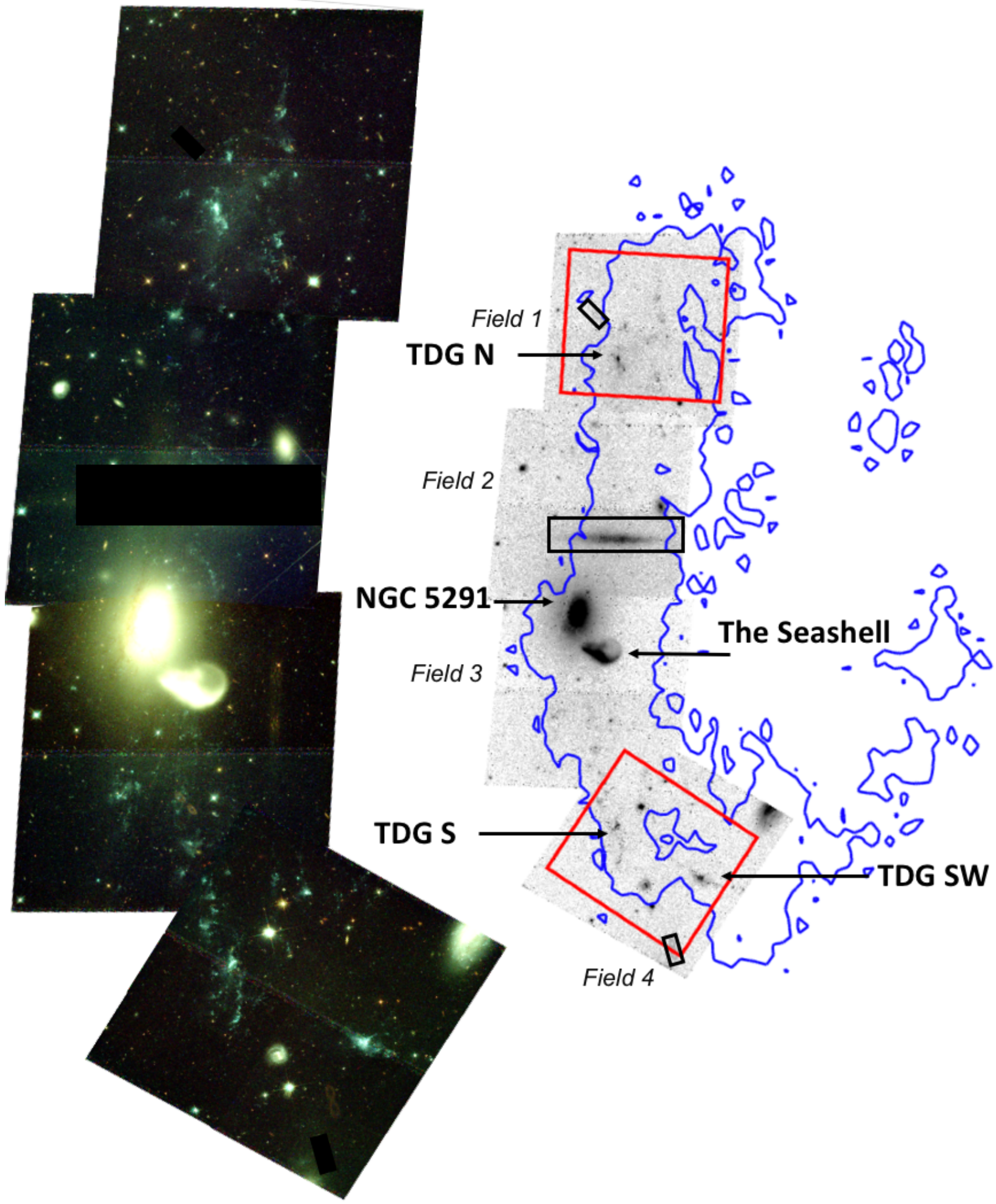

Fig. 1. Left: composite-color HST image of the system using the F475W (blue), F606W (green), and F814W (red) filters. North is up and east is to the left. Each field of view is $49.55 \mathrm{kpc} \times 53.34 \mathrm{kpc}$. Three regions contaminated by strong artifacts were masked. They are shown with black rectangles in the right image. Right: F475W image. The blue contour encircles regions where the HI column density is higher than $10^{20}$ $\mathrm{N}_{\mathrm{HI}} \mathrm{cm}^{-2}$ (VLA data, Bournaud et al. 2007). The two F160W-band fields of view are shown by the red rectangles. The central galaxy NGC 5291, the Seashell, and the three TDGs (N, S, and SW) are indicated by black arrows.

The right-hand side image shows instrument artifacts: a bright saturation shape in field 2 and the presence of figure eightshaped reflection effects in fields 1 and 4. Furthermore, a small stripe of higher noise is visible in the middle of each field of view: this is the location of the gap between the two UVIS CCDs of the camera, where we only have one exposure, and thus no cosmic ray removal. We allowed for any orientation to maximize the chance of observability. Unfortunately, the gap fell on 
Table 1. Exposure times for each field and filters in the following format: number of exposures times the time in second for a single exposure.

\begin{tabular}{lccccc}
\hline \hline & F336W & F475W & F606W & F814W & F160W \\
\hline Field 1 & $4 \times 378$ & $2 \times 368$ & $2 \times 368$ & $2 \times 368$ & $4 \times 903$ \\
Field 2 & - & $2 \times 368$ & $2 \times 368$ & $2 \times 368$ & - \\
Field 3 & - & $2 \times 368$ & $2 \times 368$ & $2 \times 368$ & - \\
Field 4 & $4 \times 378$ & $2 \times 368$ & $2 \times 368$ & $2 \times 368$ & $4 \times 903$ \\
\hline
\end{tabular}

both TDGs of field 4 . Only fields 1 and 4 are considered in the remainder of the paper. Fields 2 and 3 will be the subject of a companion paper (Fensch et al., in prep.).

We used the HST image header keyword PHOTFNU to convert image units into Jansky. All magnitude values are given according to the $\mathrm{AB}$ system in the following, unless specified otherwise.

\section{Cluster selection and photometry}

We extracted the star cluster candidates using SExtractor (Bertin \& Arnouts 1996) in the optical bands (F475W, F606W, and $\mathrm{F} 814 \mathrm{~W}$ ). The images were convolved through a Mexican hat-type filter ${ }^{1}$ with a width of two pixels to enhance the contrast with respect to the diffuse stellar light, and the detection threshold was set to $1.25 \sigma$ for at least three adjacent pixels.

We only have two exposures for each of the three optical bands (F475W, F606W, and F814W), therefore the standard pipeline cannot remove cosmic rays that are coincident on the two exposures. We proceeded to apply a first cosmic-ray subtraction by matching the location of the sources on these three filters. Only sources detected on at least both the F606W and either F475W and/or F814W images were considered for subsequent analysis. We also rejected 13 sources that were part of the Gaia DR2 (Gaia Collaboration 2018) catalog with a nonzero parallax and proper motion, which are likely foreground stars. After this step, we have 826 detections. This catalog of detections was then applied on the five bands to extract the photometry of the detected clusters.

The crowdedness of the sources in the TDGs prevented us from using a standard aperture photometry method. Instead, we used point spread function (PSF) fitting using GALFIT (Peng et al. 2002, 2010). We first removed the background light using the sigma-clipping method implemented in SExtractor. In order to remove the diffuse stellar light in the TDGs, we chose a tight mesh of $6 \times 6$ pixels, further smoothed with a $3 \times 3$ pixel kernel. The photometry was computed using PSF-fitting with GALFIT, using the PSF of the brightest unsaturated star available in the field. To avoid blending issues, we restricted the location of the peak of the PSF to vary by less than $0.08^{\prime \prime}$ compared to the center of the detection in the F606W band. Some extracted sources appeared extended and were not well fit in the F336W, F475W, F606W, and F814W bands. They were identified by a high pixel value dispersion in the residual image. The pixel size in these bands is $12 \mathrm{pc}$. In the early evolutionary stages of YMCs (1-10 Myr), the ionized gas surrounding the cluster may have a radius of around $20 \mathrm{pc}$, with a dependence on age (see, e.g., Whitmore et al. 2011). We therefore expect to have barely resolved star clusters in these bands. For 40 sources out

\footnotetext{
1 We used the filters provided in the SExtractor repository of astromatic.iap.fr.
}

of the 826 detected sources, we performed Sérsic photometry for proper subtraction. To avoid unrealistic fits, we constrained the half-light radius to be smaller than 3 pixels and a Sérsic index below 5. For consistency, we also fit the data without these constraints, and the resulting values changed only by less than half a standard deviation. These sources were not resolved in the F160W image, which has a coarser resolution, therefore we continued using PSF models for these sources. To ensure that the background subtraction method did not remove flux from either our point-like or extended sources, we verified the consistency within the error bars between the GALFIT method and an aperture photometry method for isolated sources. We used a 6-pixel radius and background estimation from the median background of eight other same-size apertures located around the source with an offset from the source of $-13,0,+13$ pixels for both the vertical and horizontal directions. Some sources were too faint for the GALFIT subtraction to converge. For these we used aperture photometry and considered this value an upper limit to the flux.

The error on the flux is obtained by combination of the Poissonian noise from the source and the removed background, the pixel-to-pixel root mean square of the background, the pixelto-pixel root mean square of the residuals, the systematic fluxdependent GALFIT flux uncertainty, and the read noise from WFC3.

A comparison of the original image and the residual after background and source subtraction is shown in Fig. 2 for the three TDGs. In this figure some small extended stellar features remain on the location of star formation complexes. These features were not considered as detections by SExtractor because of their elongated shape through the Mexican hat filter. They were also not accounted for by the background subtraction because they are smaller than the background mesh. In our subsequent analysis we restrict ourselves to sources with a signal-to-noise ratio higher than three in at least four bands, which leaves us with 439 cluster candidates.

The completeness of our star cluster candidate extraction was computed by simulating point-like sources in the image using GALFIT and testing their detection and correct flux measurement using the same analysis as described above. A simulated source was considered recovered if detected by SExtractor and if its flux was recovered within $0.3 \mathrm{mag}$ by GALFIT, as we accept a minimum signal-to-noise ratio down to 3 in a given filter. The completeness curves are shown in Fig. 3. The 95\% completeness limit is considered in the following.

\section{Deriving cluster physical properties}

\subsection{SED fitting procedure}

The set of filters we used was chosen for its ability to recover ages and extinction for young star clusters using SED fitting procedures (Anders et al. 2004). In this work, we used the SED fitting code CIGALE ${ }^{2}$ (Burgarella et al. 2005; Noll et al. 2009; Giovannoli et al. 2011; Boquien et al. 2019). This code first computes a grid of flux models for a given input of discrete parameters from the stellar models of Bruzual \& Charlot (2003), normalized to a fixed mass. In a second step, the code performs a $\chi^{2}$ analysis between the source and the flux grid, including a normalization to obtain the mass corresponding to the fit.

We chose the following range of parameters:

Star formation history. We used the Chabrier (2003) initial stellar mass function with lower and upper mass limits of 0.1

2 Code available at https://cigale.lam.fr 

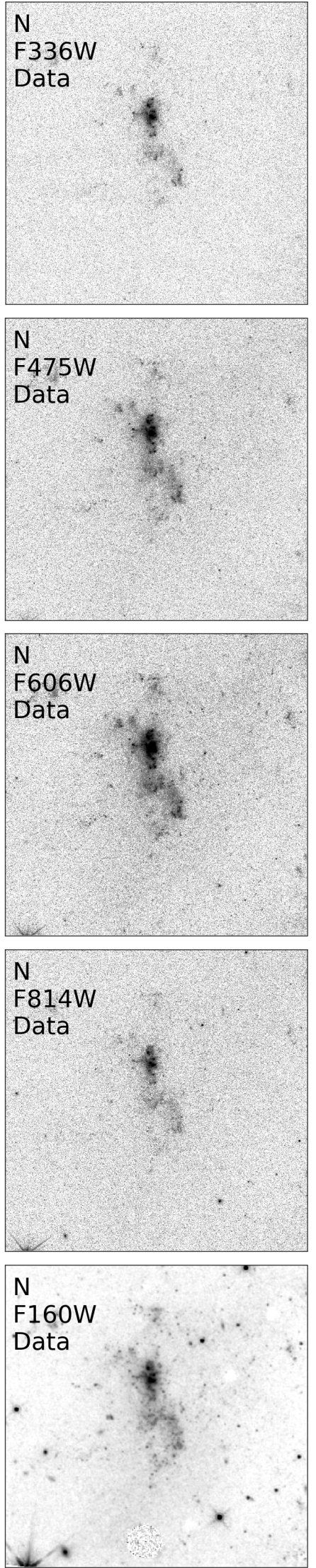
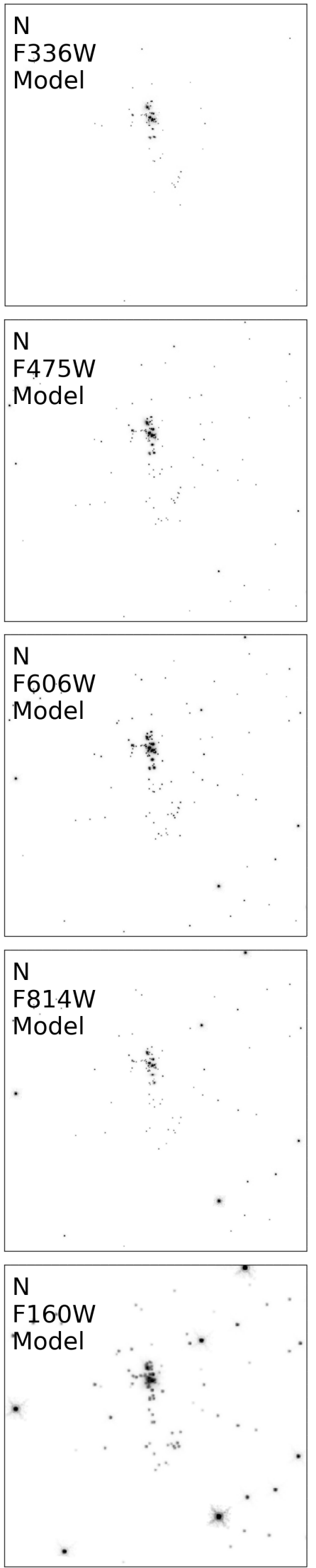
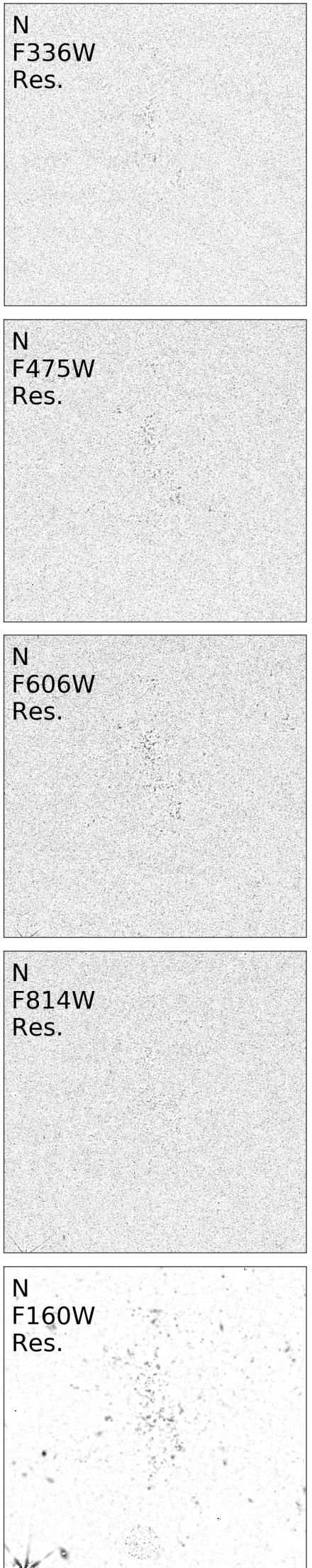

Fig. 2. Data, model, and residual images for the TDG N. The two other TDGs are shown in Appendix A. For each filter we show the data in the left column, the cluster model in the middle column, and the background-subtracted residuals in the right column. From top to bottom: F336W, F475W, F606W, F814W, and F160W. We used the L.A. Cosmics algorithm (van Dokkum 2001) to remove the cosmic rays. North is up and east is to the left. The field of view covers $14.4 \mathrm{kpc} \times 14.4 \mathrm{kpc}$. 


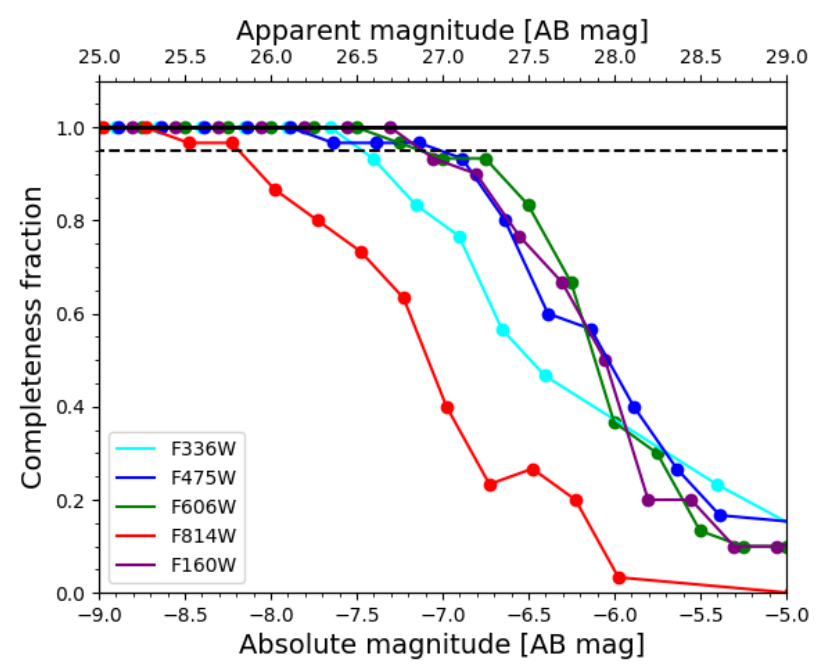

Fig. 3. Completeness curve of the star cluster detection algorithm for each filter. The horizontal dashed line at 0.95 shows the $95 \%$ completeness limit.

and $100 M_{\odot}$, respectively. We modeled our clusters as a single quasi-instantaneous burst of star formation with an exponential decay with a timescale of 0.1 Myr. To quantify the senstivity of the results on this parameter, we also modeled the star formation burst with a timescale of $1 \mathrm{Myr}$. The small variations in the resulting values are quantified in the following and do not affect our conclusions.

Age. To account for both very young star clusters and GCs, we used models from $1 \mathrm{Myr}$ to $12 \mathrm{Gyr}$. We used an adaptive spacing to account for the rapid change in the spectra at young ages. In particular, we used one model per megayear from 1 to $20 \mathrm{Myr}$ and one model per $5 \mathrm{Myr}$ from 20 to $50 \mathrm{Myr}$. The weights of the fits depend on the age grid spacing, in order to have a flat age prior.

Metallicity. The metallicity of the ring is approximately constant at around half solar metallicity (Duc \& Mirabel 1998; Fensch et al. 2016). We therefore fixed the metallicity to $Z=$ 0.008 to avoid degeneracies with age and extinction. The effect of changing the metallicity prior is discussed in the following sections.

Extinction. We used the Large Magellanic Cloud (LMC) extinction curve from Gordon et al. (2003) because it is most suitable for our half-solar metallicity. The extinction obtained from MUSE from the Balmer decrement and the LMC extinction curve gave extinction values of about $A_{V}=0.6 \pm 0.2$ mag throughout the northern TDG on a spatial scale of $180 \mathrm{pc} \times 180 \mathrm{pc}$ (Fensch et al. 2016). The NASA infrared science archive service $^{3}$ indicates a Milky Way extinction value in the line of sight of NGC 5291 of about $0.15 \mathrm{mag}$. In order to stay conservative, we allowed for extinction ranging from $A_{V}=0-2 \mathrm{mag}$.

Furthermore, we allowed the ionization parameter $\log U$ to vary between -4 and -2 with 0.5 dex steps, according to the range determined from emission line ratios with MUSE (see Fig. 10 in Fensch et al. 2016). Finally, we allowed for a fraction of escaping Lyman continuum photons between $0 \%$ and $20 \%$ (dwarfs with strong outflows have a fraction of escaping Lyman continuum photons around 15\%; see, e.g., Bik et al. 2015). We assumed a gas density of $100 \mathrm{~cm}^{-3}$ (Fensch et al. 2016).

\footnotetext{
3 https://irsa.ipac.caltech.edu/applications/DUST/
}

\subsection{Physical parameters and degeneracies}

We are interested in recovering good estimates of the ages and masses of the clusters. However, for our set of filters, there is a degeneracy between extinction and age, which is illustrated in Fig. 4. In the top panels we show see two models that fit the data well, with very different ages and extinction values. The cumulative probability density function shown in the bottom left panel shows two characteristic values for the age, and its rise is quite extended. The origin of this wide distribution is an age-extinction degeneracy: in the bottom right panel we show that both young and attenuated models and old and unattenuated models can reproduce our photometry for this particular cluster. Even though their metallicity is known, this degeneracy prevents us from deriving precise ages for all clusters.

To quantify this effect, we used a proxy for the width of the age probability distribution function (PDF) $r_{\text {age }}=F_{0.95} / F_{0.05}$, where $F_{x}$ is the age for which the cumulative age PDF reaches $x$. The extent between $F_{0.95}$ and $F_{0.05}$ is shown in the bottom left panel of Fig. 4. The particular cluster candidate shown in Fig. 4 has $r_{\text {age }}=58$. We note that emission-line information, such as $\mathrm{H} \alpha$ emission mapping at the scale of the size of a star cluster (10-20 pc), would help break this degeneracy: ionized gas would classify a given cluster as unambigously young (see, e.g., de Grijs et al. 2013). However, we cannot distinguish the star clusters on the emission-line maps we have obtained with MUSE and Fabry-Perot interferometry (see Sect. 1).

Figure 5 shows the retrieved masses and ages for the cluster candidates, where the width of their age PDF is color-coded. We note an increase in $r_{\text {age }}$ with age, which is due to the slower spectral evolution with age, and also a significant number sources for which $r_{\text {age }}>50$, which might be subject to degeneracies. In this study we are not interested in old GCs. We therefore only consider clusters with retrieved age below 3 Gyr in Fig. 5. Older clusters, for which the fixed half-solar metallicity prior is not adapted for mass and age determination will be presented in a companion paper, along with the study of fields 2 and 3 (Fensch et al., in prep.).

Figure 5 also shows the completeness limit as obtained in Sect. 3. These curves were obtained from the flux models computed by CIGALE. For each age, the curves show the minimum mass for which a cluster would have a $95 \%$ probability to be detected with a signal-to-noise ratio $(\mathrm{S} / \mathrm{N})$ above 3 in at least four bands. We show the completeness curves for two assumed extinctions, $A_{V}=0$ and $1.1 \mathrm{mag}$. This latter value was the maximum extinction obtained using the Balmer decrement from the field of the northern TDG in Fensch et al. (2016). Based on this figure, we assume that our sample is complete for clusters younger than $30 \mathrm{Myr}$ above a mass of $1.5 \times 10^{4} M_{\odot}$.

\subsection{Young cluster mass function}

In the following we explore the properties of the young clusters. In order to have a sufficient number of detections, we chose to consider only clusters younger than $30 \mathrm{Myr}$.

Because of the degeneracy effect, no complete sample can be compiled. Young and strongly attenuated clusters could in principle masquerade as old and unattenuated clusters. To define our sample, we used the shape of the age PDF. In particular, we write

$P[$ age $<X]=\int_{0}^{X} \operatorname{PDF}(t) \mathrm{d} t$,

with $X$ in megayears, the probability that the cluster candidate is younger than $X$ Myr. 

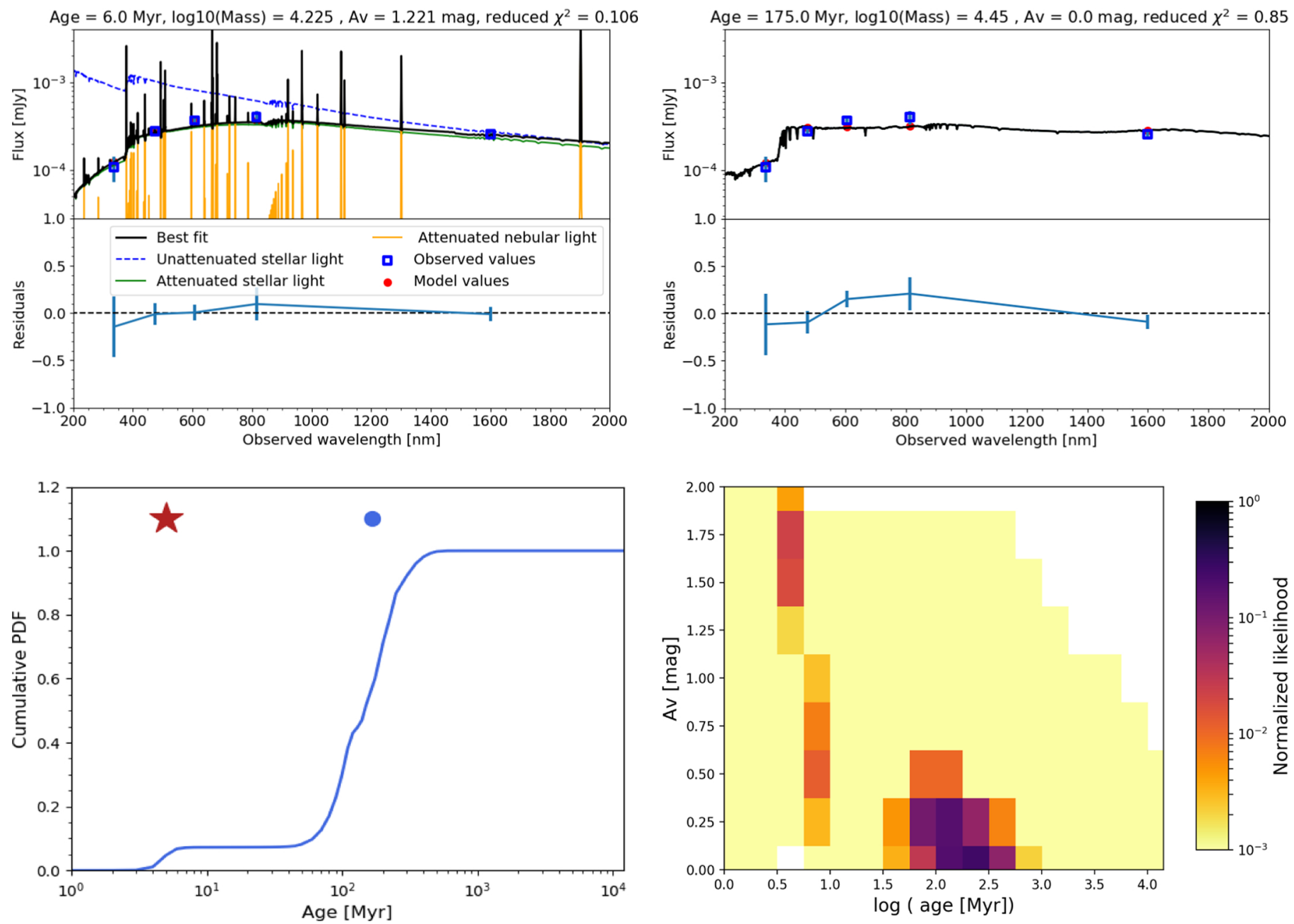

Fig. 4. Top left: example of the best fit for one cluster candidate. The retrieved physical parameters and the reduced $\chi^{2}$ are given at the top of the plot. Top right: best fit for the same cluster candidate when $A_{V}=0$ mag is imposed. Bottom left: cumulative age PDF for the cluster. The star shows the best-fitting age. The blue dot shows the output value of CIGALE. Bottom right: normalized likelihood distribution for age and extinction for the given cluster candidate.

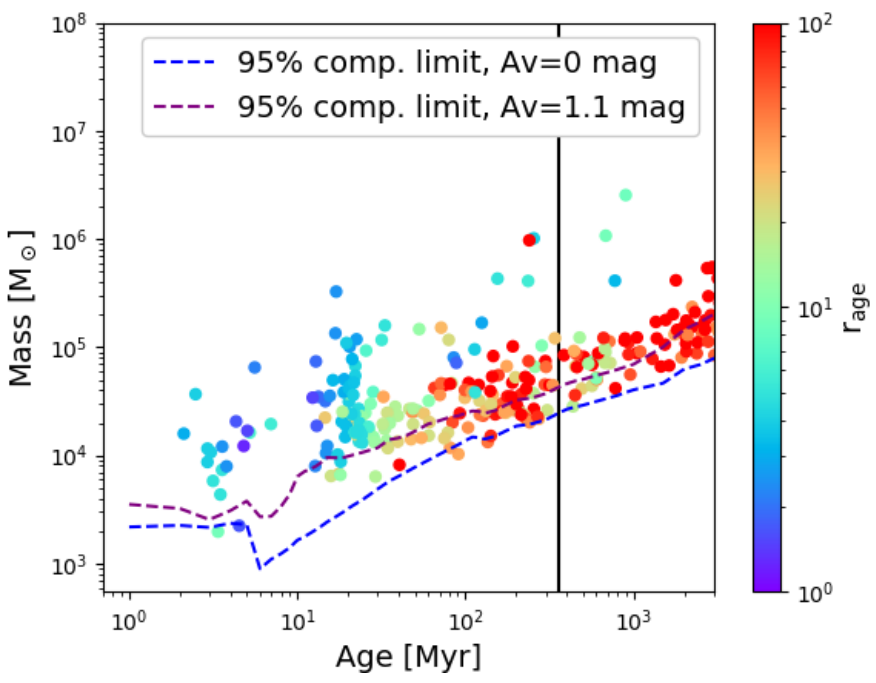

Fig. 5. Estimated mass-age distribution for the cluster candidates. The color indicates the width of the age PDF, as defined in the text. The two dashed lines indicates the $95 \%$ completeness limit in the age-mass plane, assuming a given extinction. The black line shows the estimated time of the interaction that created this system (see text).
In the following, we define our young cluster sample by adopting $P$ [age $<40$ ] $>0.5$ and the modal value of the age PDF that is enclosed in [1 Myr, $30 \mathrm{Myr}$ ].

We used $40 \mathrm{Myr}$ as the upper bound for the PDF integral because a limit of $30 \mathrm{Myr}$ rejected clusters with ages between 20 and $30 \mathrm{Myr}$, for which a large portion of their PDFs extends beyond $30 \mathrm{Myr}$. We then chose a higher upper bound for the integral calculation, and used the condition on the mode of the PDF to ensure that the highest likelihood was still reached within the $[0,30]$ Myr interval. To quantify this effect, we used this sample selection in association with the spectral models created by CIGALE with input ages between 20 and 40 Myr. Only $28 \%$ of the clusters with an input age in the range [20,30] Myr were included in our sample when we used $30 \mathrm{Myr}$ as upper bound, while this fraction rose to $71 \%$ for $40 \mathrm{Myr}$ as upper bound. The contamination fraction, that is, the fraction of models assigned to the sample that have ages in $(30,40] \mathrm{Myr}$, is $0 \%$ in the first case and $6 \%$ in the second case. A limit of $40 \mathrm{Myr}$ instead of $30 \mathrm{Myr}$ in the definition of our sample therefore better represents the clusters that are genuinely younger than $30 \mathrm{Myr}$.

We discuss in Appendix B two other sample selections: a secure sample, defined by $P$ [age $<40]>0.9$, and an inclusive sample defined by $P[$ age $<40]>0.1$. The exact same analysis was performed on these two samples for comparison purposes. 


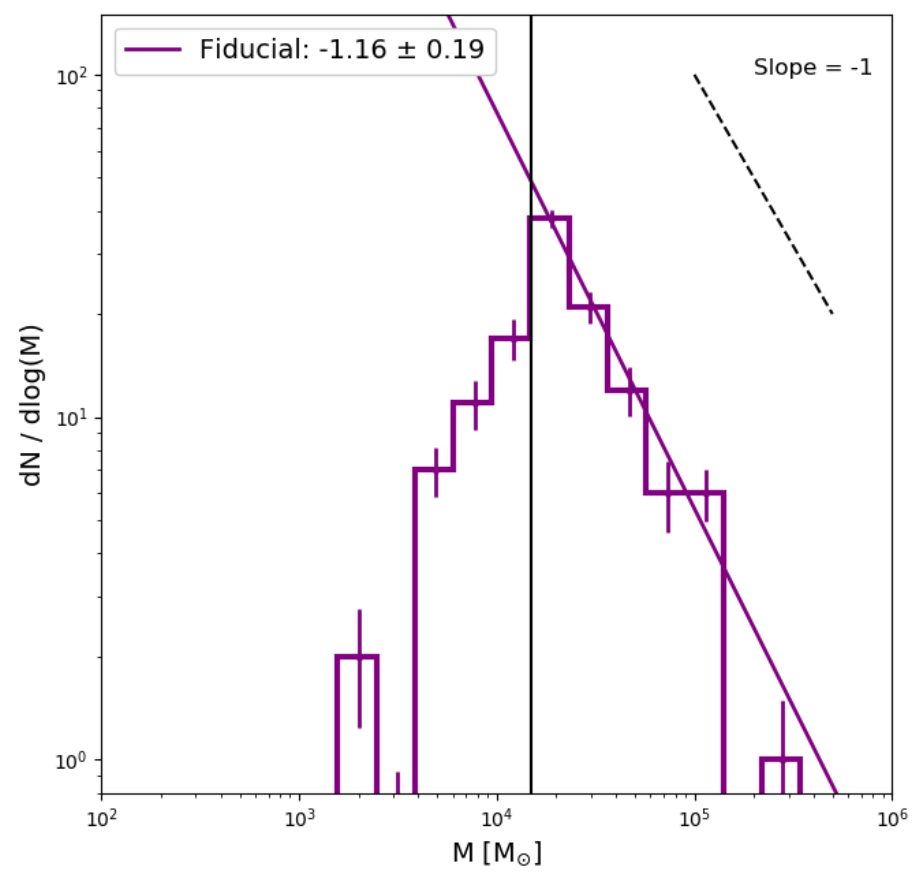

Fig. 6. CMFs for the young cluster sample described in the text. The power-law fit is determined for bins with masses higher than $1.5 \times 10^{4} M_{\odot}$, shown by the black vertical line. The legend shows the slope and uncertainty of the corresponding fit.

Because the first selection is very restrictive and the other includes clusters that are too old, this additional analysis gives an idea of the strict boundaries within which our result may vary.

Figure 6 shows the cluster mass function (CMF) for our young cluster sample. A power-law fit to the diagram for bins more massive than $1.5 \times 10^{4} M_{\odot}$ gives a slope of $-1.16 \pm$ 0.19 for the evolution of $\frac{\mathrm{d} N}{\mathrm{~d} \log M}$ with $M$. This gives $\frac{\mathrm{d} N}{\mathrm{~d} M}=$ $\frac{\mathrm{d} N}{M \mathrm{~d} \log M} \propto M^{\alpha}$, with $\alpha=-2.16 \pm 0.19$. The values obtained for the lower metallicity prior $(Z=0.004)$ and for the $1 \mathrm{Myr}$ timescale are consistent within the one-sigma uncertainty. The obtained mass distribution is consistent with a mass distribution decreasing with a power slope of $\alpha \sim-2$, as many other studies of young star cluster formation have shown (see, e.g., Portegies Zwart et al. 2010). This suggests that the formation of star clusters in the gas ring and TDGs occurs in a similar fashion to that of the other studied environments. This can be interpreted as a legacy of the hierarchical collapse of gas clouds (see, e.g., Elmegreen \& Efremov 1997).

\subsection{Star cluster formation efficiency in the TDGs}

The cluster formation efficiency (CFE) of galaxies can be characterized by $\frac{\mathrm{CFR}}{\mathrm{SFR}}$, where CFR is the cluster formation rate (in $\left.M_{\odot} \mathrm{yr}^{-1}\right)$. It has been argued that galaxies follow a power-law relation with positive index in the CFE and the SFR surface density $\left(\Sigma_{\text {SFR }}\right)$ plane (Larsen \& Richtler 2000; Billett et al. 2002; Goddard et al. 2010). A similar relation was derived on theoretical grounds by Kruijssen (2012). However, Chandar et al. (2017) claimed that the former empirical relation was driven by an underestimation of the CFR of both the LMC and the Small Magellanic Cloud (SMC) due to an inconsistent age range selection. In contrast, they found a constant value of the CFE of $24 \% \pm 9 \%$, independent of $\Sigma_{\mathrm{SFR}}$.

In order to compute the CFE for our system, we constructed our young cluster samples based on the same definition as above,

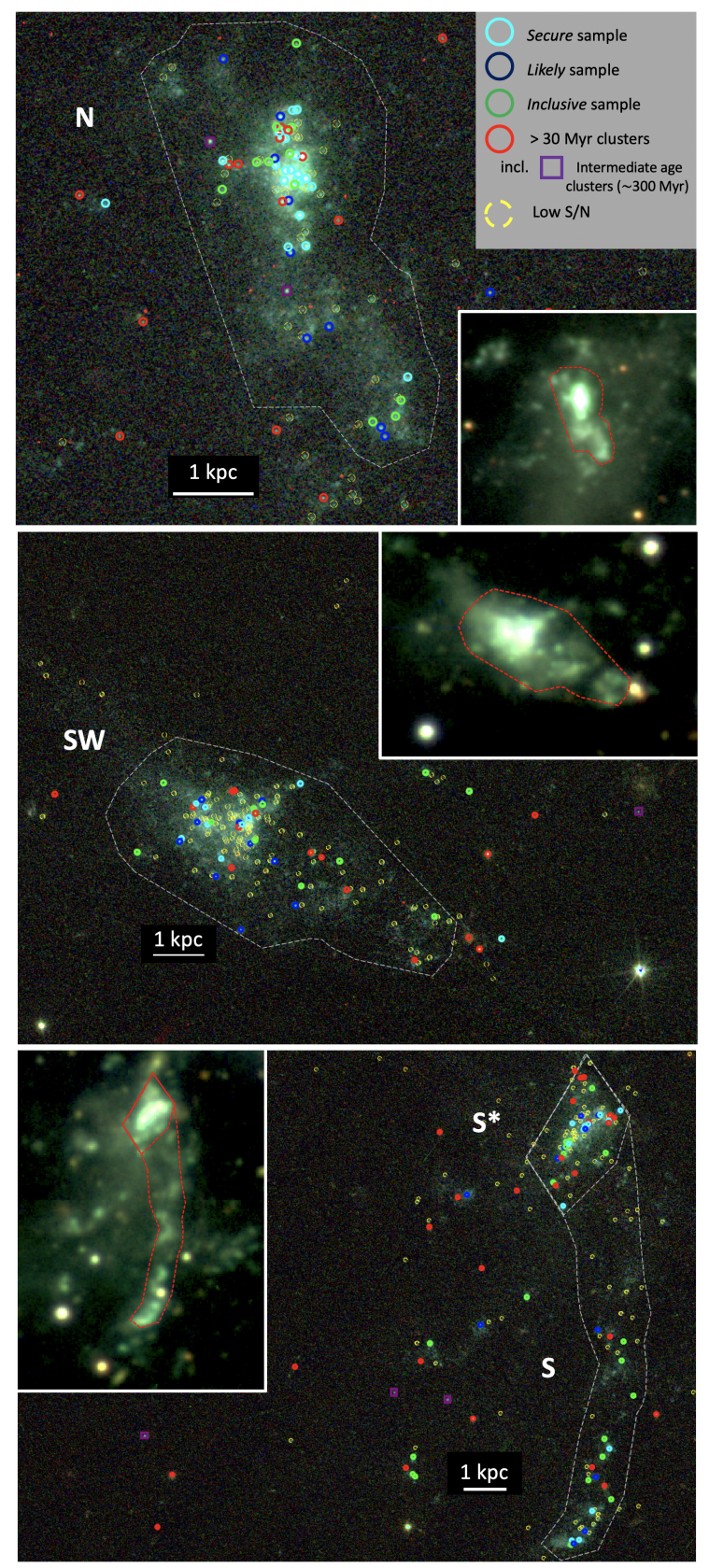

Fig. 7. True-color image of the three TDGs: NGC 5291N (top panel), NGC 5291SW (middle panel), and NGC5291S (bottom panel). The definitions of the young sample, degenerate clusters, and the sample older than $30 \mathrm{Myr}$ are given in the text. The intermediate clusters are part of the sample older than $30 \mathrm{Myr}$ and are discussed in Sect. 4.6. We also show detected clusters that do not have $S / N>3$ in at least four bands as the low $\mathrm{S} / \mathrm{N}$ sample. The dashed white contours show the area considered to compute $\Sigma_{\mathrm{SFR}}$. Bottom panel: two contours that are explained in the text. The inset shows a VRI image from FORS (Fensch et al. 2016), with the same contours. Only clusters inside the white contours are considered for the computation of the CFE.

but for each of the three TDGs. In order to limit the effects of degeneracies, we used as a minimum value for the fitting prior $A_{V}>0.3 \mathrm{mag}$, which is justified by the extinction maps obtained with MUSE by Fensch et al. (2016). We show the location of our young cluster sample in the three TDGs in Fig. 7. We also show the detections that are degenerate and securely old (see definition in Sect. 4.3). We also considered a smaller star cluster subsample for the $\mathrm{S}$ dwarf, shown in Fig. 7, which we call $\mathrm{S}^{*}$ 
Table 2. SFR, area, and CFE for the TDGs.

\begin{tabular}{lccccc}
\hline \hline Galaxy & $\begin{array}{c}\text { SFR } \\
{\left[M_{\odot} \mathrm{yr}^{-1}\right]}\end{array}$ & $\begin{array}{c}\text { Area } \\
{\left[\mathrm{kpc}^{2}\right]}\end{array}$ & $\begin{array}{c}\text { CFE } \\
{[\%]}\end{array}$ & $\begin{array}{c}\mathrm{K} 12 \\
{[\%]}\end{array}$ & $\begin{array}{c}\mathrm{J} 16 \\
{[\%]}\end{array}$ \\
\hline TDG N & $0.19 \pm 0.06$ & 12.76 & $47_{-21}^{+21}$ & $14_{-2}^{+2}$ & $22_{-9}^{+10}$ \\
TDG SW & $0.14 \pm 0.05$ & 17.44 & $33_{-16}^{+17}$ & $10_{-2}^{+2}$ & $15_{-7}^{+8}$ \\
TDG S & $0.12 \pm 0.03$ & 17.17 & $45_{-15}^{+16}$ & $10_{-1}^{+1}$ & $14_{-6}^{+8}$ \\
Including S* & $0.08 \pm 0.03$ & 4.59 & $60_{-26}^{+26}$ & $15_{-3}^{+2}$ & $23_{-9}^{+11}$ \\
\hline
\end{tabular}

Notes. The last two columns show the expectation of the CFE from the Kruijssen (2012) and Johnson et al. (2016) models for the measured SFR surface density, labeled K12 and J16.

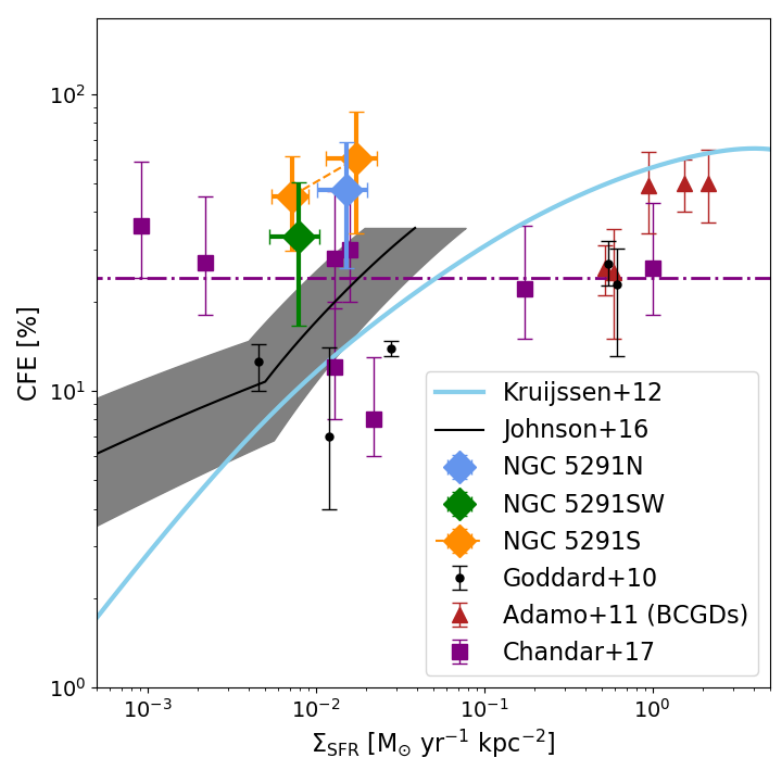

Fig. 8. Distribution of our TDGs in the CFE- $\Sigma_{\text {SFR }}$ plane. For the TDG NGC 5291S we show two points, the full TDG (with lower CFE and $\left.\Sigma_{\mathrm{SFR}}\right)$ and only $\mathrm{S}^{*}$. The dataset of Goddard et al. (2010) is shown in black. The sample of Chandar et al. (2017) and their fit to their data is shown in purple. For the SMC and the LMC we only show the values computed by the latter reference (see text). In red are shown the BCDGs of Adamo et al. (2011). The continuous blue line shows the prediction of the model by Kruijssen (2012) (see text). The gray band shows a modified version of this model using the Bigiel et al. (2008) relation (Johnson et al. 2016).

in the following. This is motivated by the elongated shape of this TDG, which suggests that TDG 5291S might be composed of two distinct objects, despite the apparent coherent HI rotation (Lelli et al. 2015).

Following previous studies on the CFE (see, e.g., Goddard et al. 2010; Adamo et al. 2011), we used the CMF to infer the total mass in clusters down to $10^{2} M_{\odot}$ by forcing a canonical power-law shape $\frac{\mathrm{d} N}{\mathrm{~d} M} \propto M^{\alpha}$, with $\alpha=-2$ fit to the histogram. This fixed index of -2 was also used in the studies we refer to in this section, and is also supported by the shape of the CMF covering the full cluster sample (see Fig. 6). The SFR was obtained from the $\mathrm{H} \alpha$ (Boquien et al. 2007), which used a Salpeter (1955) IMF, whereas our mass estimates were obtained using a Chabrier (2003) IMF. We therefore multiplied the SFR obtained using the $\mathrm{H} \alpha$ by Boquien et al. (2007) by a factor 0.70 to account for the different flux ratio of SFR to $\mathrm{H} \alpha$ obtained for the two IMFs (see, e.g., Kennicutt et al. 2009). We also corrected the SFR for the mean extinction measured in
Table 3. Significance, in standard deviations, of the offset of the data points compared to the three relations: Kruijssen (2012), Johnson et al. (2016), and Chandar et al. (2017), K12, J16, and C17.

\begin{tabular}{lccc}
\hline \hline Galaxy & K12 & $\mathrm{J} 16$ & $\mathrm{C} 17$ \\
\hline TDG N & 1.6 & 1.1 & 1.0 \\
TDG SW & 1.3 & 1.0 & 0.5 \\
TDG S & 2.2 & 1.7 & 1.1 \\
Including S* & 1.7 & 1.3 & 1.3 \\
TDG N+SW+S & 3.8 & 3.1 & 2.5 \\
TDG N+SW+S* & 3.5 & 2.8 & 2.6 \\
\hline
\end{tabular}

TDG N, $A_{V}=0.6 \mathrm{mag}$ (Fensch et al. 2016). The obtained values of the CFE are summarized in Table 2. The lower metallicity prior $(Z=0.004)$ gives consistent results within the one-sigma uncertainty and changes the CFE values by less than $10 \%$. The $1 \mathrm{Myr}$ star formation timescale changes the CFE values by less than $3 \%$.

To compare the TDGs with other star cluster forming galaxies, we place these values in the CFE- $\Sigma_{\mathrm{SFR}}$ plane in Fig. 8. The TDGs are located in the same regime as the BCDGs, with CFE above $45 \%$ for TDG $\mathrm{N}$ and TDG S. They are located systematically above the empirical Chandar et al. (2017) relation, but are consistent within 0.5 to $1.3 \sigma$.

In Fig. 8 we show the current model and empirical predictions. The blue curve shows the model ${ }^{4}$ by Kruijssen (2012, $\mathrm{K} 12$ in the following) for a gas velocity dispersion of $30 \mathrm{~km} \mathrm{~s}^{-1}$. We also show the version of the model calibrated with the Bigiel et al. (2008) relation between the SFR and the gas density (Johnson et al. 2016, J16). In purple we show the universal value of $24 \%$ suggested by Chandar et al. (2017, C17). The computed CFEs are systematically above these three relations. The significance of this deviation for the TDGs and the full system is measured with random draws of relation and data values, assuming Gaussian distributions. For the combined TDGs, this is equivalent to multiplying the probabilities that the CFE of each TDG has to be compatible with the relation. The significances are given in Table 3. While the measurement of each TDGs differs by less than $2.1 \sigma$ of each relation, the combination of the TDG N, SW, and $\mathrm{S}$ is above these relations by $3.8 \sigma$ for $\mathrm{K} 12,3 \sigma$ for $\mathrm{J} 16$, and $2.5 \sigma$ for $\mathrm{C} 17$. These numbers slightly change when $\mathrm{S}^{*}$ is used instead of $\mathrm{S}$ in the sample. Our sample of TDGs is then significantly above the current model and empirical relations.

We used an age range [1-30] Myr, which is broader than that typically used in these studies $([1-10] \mathrm{Myr})$. We chose this range because there were not enough clusters younger than $10 \mathrm{Myr}$ to properly measure the CFE. We did not correct for the mass evolution or destruction that may have happened, in particular cluster disruption by gas removal (infant mortality, Boutloukos \& Lamers 2003; Whitmore et al. 2007), which has a timescale of 10-40 Myr (Kroupa \& Boily 2002; Fall et al. 2005; Goodwin \& Bastian 2006). This means that we are missing clusters that have been disrupted and mass that has been lost from the detected clusters. The fact that we did not correct for this effect suggests that we might be underestimating the CFE of our TDGs (see discussion in $\mathrm{C} 17$ ). Finally, we note that at the distance of NGC 5291, the data are contaminated by young star associations that are unbound and did not have time to dissolve (see, e.g., Messa et al. 2018). This unresolved process might lead to an overestimation of the computed CFE.

\footnotetext{
4 Model accessible at: https://wwwmpa.mpa-garching.mpg.de/ $\mathrm{cfe} /$. We used the integrated CFE model.
} 


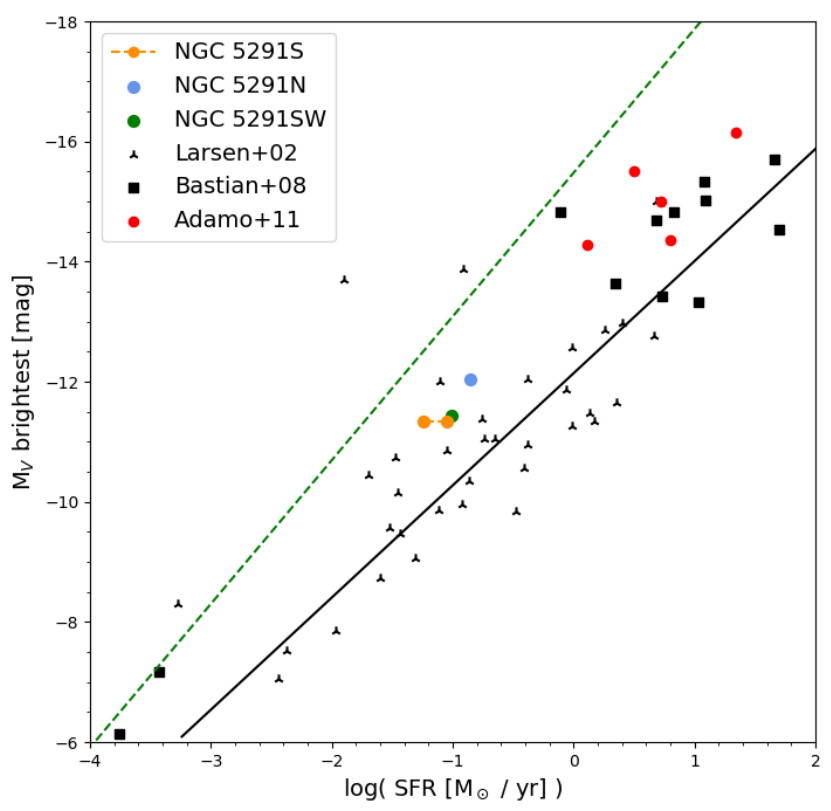

Fig. 9. Brightest cluster $M_{V}-\mathrm{SFR}$ relation for different galaxy samples. The NGC 5291S data point with the lowest SFR shows the value for $S^{*}$ only. The black line shows the fit of the Larsen (2002) sample. The dashed green line shows the maximum $M_{V}$ expected for a given SFR if all the star formation occurs in clusters with a $\frac{\mathrm{d} N}{\mathrm{~d} M} \propto M^{-2}$ power law (Bastian 2008).

As explained in Sect. 4.2, we also performed the same analysis on two different cluster samples with more restrictive or more relaxed age constraints. The analysis is presented in Appendix B. In particular, we introduce the secure sample, which only contains clusters that are almost not affected by degeneracies and have a narrow age PDF, thus underestimating the genuine sample of clusters younger than $30 \mathrm{Myr}$. For this sample, the sample of TDGs $(\mathrm{N}, \mathrm{SW}, \mathrm{S})$ is above the relations by $2.8 \sigma$ for $\mathrm{K} 12,1.8 \sigma$ for $\mathrm{J} 16$ and $1 \sigma$ for $\mathrm{C} 17$. The fact that the $\mathrm{CFE}$ of the full sample is above the model relations of $\mathrm{K} 12$ by $2.8 \sigma$ confirms that this mismatch is robust against the age selection procedure. However, the CFE of the full sample of TDGs is only $1.8 \sigma$ from the $\mathrm{J} 16$ relation and is consistent with the $\mathrm{C} 17$ relation within $1 \sigma$. The combination of the CFEs of the TDGs are thus not statistically significantly above these two relations when this restricted sample alone is considered.

Finally, we combined bands with different PSF. We added the F160W as it provides a good filter combination to reduce degeneracies (Anders et al. 2004). However, the coarser spatial resolution of the F160W might lead to an overestimation of the photometry in this band (see, e.g., Bastian et al. 2014, but with aperture photometry). This effect is discussed in Sect. 5.1.

\subsection{Brightest cluster - SFR relation}

Larsen (2002) found a positive correlation between the $V$-band absolute magnitude $\left(M_{V}\right)$ of the brightest cluster versus the SFR of the host, which was interpreted as a size-of-sample effect: the higher the SFR, the more clusters and thus the more likely high-mass clusters would be found. The location of the three TDGs and other cluster-forming systems in the $M_{V}-$ SFR plane is shown in Fig. 9. Our three TDGs are located within the intrinsic scatter of the relation of Larsen (2002). This suggests that the magnitude of the brightest cluster is a good tracer of the SFR for these systems, similar to what has generally been observed for star-forming galaxies.

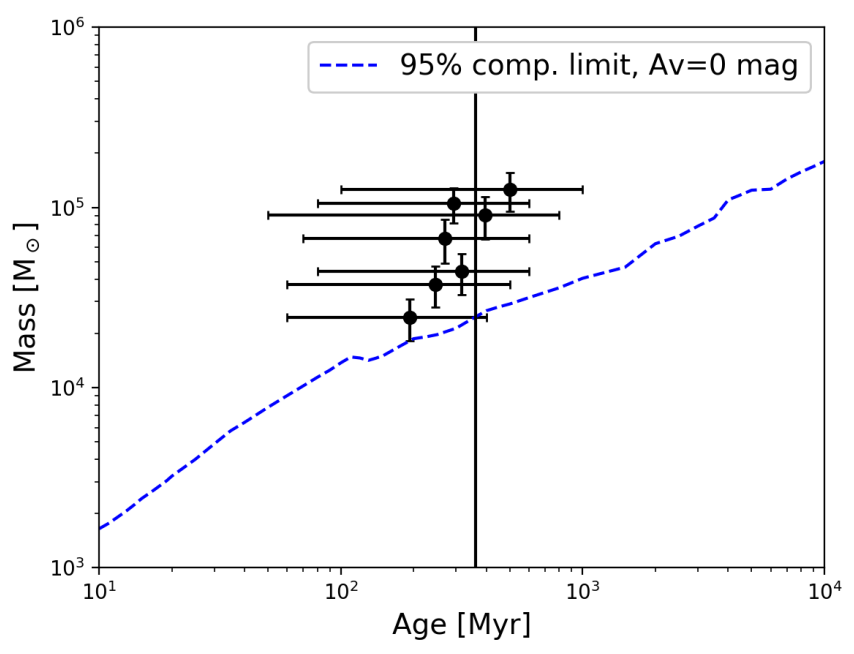

Fig. 10. Masses and ages of our conservative sample of intermediateage star clusters. The $x$-axis error bar shows the width between the first and last decile of the age PDF. The $y$-axis error bar shows the standard deviation for the mass estimate. The vertical black line shows the formation time of the ring structure, $\sim 360 \mathrm{Myr}$, as determined by Bournaud et al. (2007).

Table 4. CFE values for the three TDGs, without the F160W band (second column) and significance in standard deviations of the offset of the data with respect to the models (three last columns).

\begin{tabular}{lcccc}
\hline \hline Galaxy & CFE [\%] & K12 & J16 & C17 \\
\hline TDG N & $37_{-15}^{+15}$ & 1.5 & 0.8 & 0.7 \\
TDG SW & $26_{-10}^{+11}$ & 1.4 & 0.8 & 0.1 \\
TDG S & $33_{-14}^{+14}$ & 1.6 & 1.2 & 0.5 \\
including S* & $46_{-25}^{+25}$ & 1.2 & 0.8 & 0.8 \\
TDG N+SW+S & - & 3.5 & 2.6 & 1.9 \\
TDG N+SW+S* & - & 3.3 & 2.4 & 2.0 \\
\hline
\end{tabular}

\subsection{Presence of intermediate-age clusters}

It is interesting to study whether the peculiar environment of NGC 5291 may allow for the survival of clusters over timescales of $\simeq 100$ Myr. Bournaud et al. (2007) estimated that the interaction that triggered the formation of the ring occurred around $360 \mathrm{Myr}$ ago. We showed in Sect. 4.2 and Fig. 5 that an age cannot be estimated with high precision. In Fig. 5 we show that some cluster candidates with an estimated age between 100 and 2000 Myr have a relatively low $r_{\text {age }}$ for the inferred age, that is, they are younger than $30 \mathrm{Myr}$. A high PDF is expected around these ages because the stellar spectrum does not change much in this part of the stellar evolution period. Some of these clusters might therefore have formed at the formation time of the ring and survived for several $100 \mathrm{Myr}$ in this environment.

To construct a conservative sample of candidates with intermediate ages, we first selected clusters with $P[50<$ age $<$ $2000]>0.9$. We chose an upper limit of 2000 Myr because the age PDFs can be quite extended for this age range (see Fig. 5). We note that this selection does not change when we allow for an extended star formation history with an exponential decrease timescale of 1 or $5 \mathrm{Myr}$, compared with our fiducial value of $0.1 \mathrm{Myr}$, which was chosen to model a quasi-instantaneous burst.

Moreover, we ensured that the photometry of these clusters is not consistent with them being old metal-poor or metalrich GCs from the GC system of NGC 5291. For this, we ran 


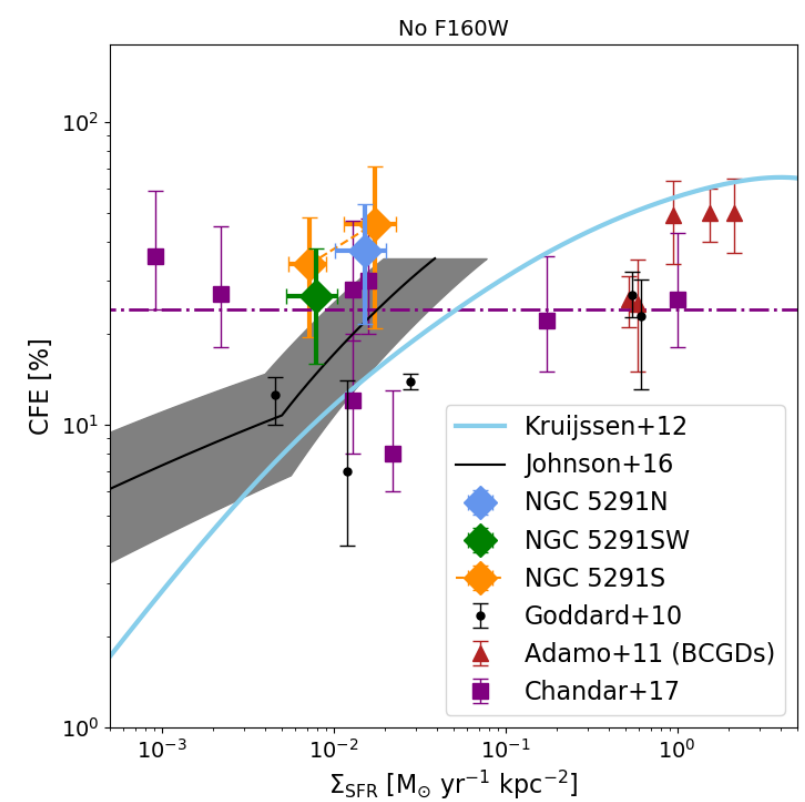

Fig. 11. Same legend as Fig. 8. The analysis was made without considering the F160W band.

CIGALE with a broader metallicity prior ( $Z$ can be 0.0004 , 0.004 , or 0.008 , instead of only 0.008 ), and we ruled out clusters for which $P$ [age $>2500]>0.1$. We ended up with seven clusters. Their ages and masses are shown in Fig. 10. Their mass range is between $2 \times 10^{4}$ and $2 \times 10^{5} M_{\odot}$. Their location is shown with purple squares in Fig. 7 . Three are located close to the TDG $\mathrm{N}$, one is close to TDG SW, and three are close to TDG S.

Clusters with similar masses and ages have previously been observed in a number of dwarf galaxies (see, e.g., Larsen et al. 2004; de Grijs et al. 2013). However, their presence in NGC 5291 shows that massive star clusters can survive the very turbulent and gaseous environment of a tidal dwarf galaxy from its formation up to hundreds of million years.

\section{Discussion}

\subsection{Effect of including the $F 160 \mathrm{~W}$ band}

In Sect. 4.4 we showed that two TDGs, $\mathrm{N}$ and $\mathrm{S}$, have very high CFEs, with an average value of $42 \%$. While it has been argued on theoretical grounds that star cluster formation should be more efficient in low-metallicity environments, all other factors being equal (Peebles 1984; Kimm et al. 2016), these metal-rich TDGs reach a similar CFE as metal-poor BCDGs.

As pointed out in Sect. 4.4, the estimated masses could have been affected by an overestimation of the flux in the F160W band. We included the F160W filter to reduce the degeneracies on the estimation of the age and mass of the clusters (Anders et al. 2004). However, this band has a coarser spatial resolution than the four others. The F160W band PSF has an FWHM of about $0.18^{\prime \prime}$, while the four other bands have PSF FWHMs of $0.06^{\prime \prime}$. Some F160W flux measurements might have been contaminated by regions that are very close to the clusters and that are not included in the other bands, which might affect the derivation of the physical quantities (e.g., Bastian et al. 2014, in the case of aperture photometry).

To test the effect of adding the F160W band, we removed it from the analysis for the measurement of the CFE. The CFEs we obtained are summarized in Table 4. They are lower than those obtained with the five bands by typically $22 \%$.

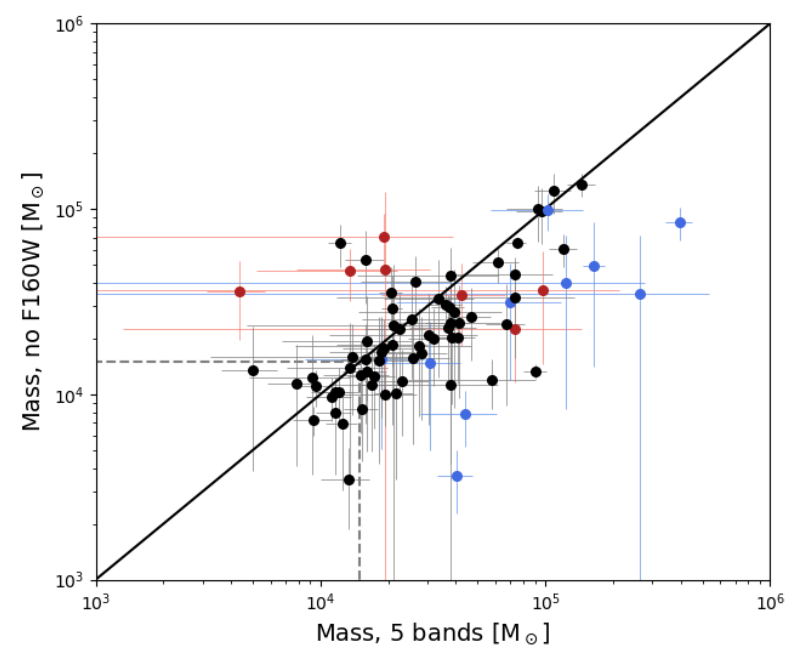

Fig. 12. Comparison between the mass estimation between the analysis including or excluding the F160W band. The blue points show clusters that are included as younger than $30 \mathrm{Myr}$ only in the analysis without F160W. The red points show clusters that are included in this category only when the F160W band is included. The thick line shows the identity function. The two dashed lines show the mass completeness limit.

In Fig. 12 we show the change in the mass estimation of the clusters that are considered as younger than $30 \mathrm{Myr}$ and located in the TDGs. Of the clusters we that determined to be younger than $30 \mathrm{Myr}$, a few are estimated to be older than this limit when the F160W filter is excluded (five are higher than the completeness limit) while others are estimated to be younger with the F160W band (eight above the limit). Moreover, while the estimated masses are very similar, there is a trend toward lower masses when the F160W band is excluded.

Figure 11 shows that even without the F160W band, the CFEs of the TDGs are systemically above the three relations from the literature. The significance of the discrepancies is computed in Table 4. The full sample of TDGs (N, SW, and S) is $3.5 \sigma$ and $2.6 \sigma$ above the $\mathrm{K} 12$ and $\mathrm{J} 16$ relations, respectively, that is, with a certainty higher than $99.5 \%$. However, the offset with the $\mathrm{C} 17$ relation is only $1.9 \sigma$. Thus, the exclusion of the F160W reduces the discrepancy between the data and the relations from the literature, which becomes statistically insignificant only for $\mathrm{C} 17(<2.5 \sigma)$. This suggests that the discrepancy to the three relations from the literature is robust over the possible overestimation of the F160W band due to a coarser spatial resolution.

\subsection{Origin of the high cluster formation efficiency}

We showed in Sect. 4.4 that our sample of TDGs has high CFEs, above $30 \%$, similar to what is observed for BCDGs. While it has been argued on theoretical grounds that star cluster formation should be more efficient in low-metallicity environments, all other factors being equal (Peebles 1984; Kimm et al. 2016), these metal-rich TDGs reach a similar CFE as metal-poor BCDGs.

It is interesting to note that all galaxies from the literature whose CFE is higher than $20 \%$ are galaxies involved in an interaction of some sort, with the exception of the center of M 83 in the sample of Goddard et al. (2010). It is also interesting to note that the late stages of mergers, while leading to similar $\Sigma_{\mathrm{SFR}}$ as the early stages, trigger the formation of only a few clusters (Renaud et al. 2015), and therefore have a much lower CFE. We interpret this to mean that cluster formation is triggered by the onset of compressive turbulence that is triggered mainly during the early times of galaxy interaction. 
Although the TDGs in NGC 5291 were not formed in bona fide merging galaxies, they are located in a gas-dominated environment and are probably not yet fully relaxed. It is thus possible that their dynamical state, in terms of compressive turbulence, is similar to that of interacting galaxies, possibly because of accretion from the gas ring (Fensch et al. 2016).

\subsection{Evolution of the star cluster system}

We showed in Sect. 4.6 that some clusters could survive their birth environment for several $100 \mathrm{Myr}$. The fact that we could find some in the gaseous ring shows that we can expect the survival of massive star clusters from the formation of the tidal dwarf galaxy to at least several $100 \mathrm{Myr}$.

The evolution of this specific star cluster system in the future is an open question. In the following, we consider that our clusters survived after gas expulsion and we do not consider their infant mortality rate, which is arises because the internal feedback expels the gas and destabilizes the cluster. This rate has a timescale of 10-40 Myr (see Sect. 4.3). We modeled the mass loss due to cluster evaporation during its relaxation as $\Delta M(t)=\mu_{\mathrm{ev}} t$, with $\mu_{\mathrm{ev}}$ the evaporation rate (Hénon 1961; Fall \& Zhang 2001; Jordán et al. 2007), which is given by

$\mu_{\mathrm{ev}}=345 M_{\odot} \mathrm{Gyr}^{-1}\left(\frac{\rho}{M_{\odot} \mathrm{pc}^{-3}}\right)^{1 / 2}$,

where $\rho=3 M /\left(8 \pi R_{\mathrm{eff}}^{3}\right)$ is the half-mass density of the cluster. $M$ and $R_{\text {eff }}$ are its mass and half-mass radius. This is a likely lower limit to the genuine evaporation rate of stellar clusters because it does not include the effects of stellar evolution, gas cloud encounters, and tidal effects from the host TDG.

The typical density of YMCs is $10^{3} M_{\odot} \mathrm{pc}^{-3}$ (see review by Portegies Zwart et al. 2010). For this density, we obtain $\mu_{\mathrm{ev}} \sim$ $10^{4} M_{\odot} \mathrm{Gyr}^{-1}$. Under this hypothesis, we may conclude that most of the stellar clusters of our system will be destroyed in a few gigayears at most, at least by internal relaxation.

Now we consider a cluster with a mass of $2 \times 10^{4} M_{\odot}$. Reaching the typical density of YMCs implies a typical half-mass radius of $1.3 \mathrm{pc}$. As one pixel corresponds to a physical size of $12 \mathrm{pc}(36 \mathrm{pc}$ for the F160W band) at the distance of NGC 5291, we cannot constrain the size of our clusters. Most of our sources are well fit by a PSF, which means a half-mass radius securely below $6 \mathrm{pc}$. The few sources that are not well fit by a PSF (see Sect. 3) have halflight radii that can reach up to 2.5 pixels. However, they could also be blended detections or extended nebular emission from the ionized outskirts of young clusters. For a mass of $2 \times 10^{4} M_{\odot}$ and a half-mass radius of $6 \mathrm{pc}$, we obtain $\mu_{\mathrm{ev}} \sim 10^{3} M_{\odot} \mathrm{Gyr}^{-1}$. The timescale for the destruction, considering only the effects of relaxation, is therefore a Hubble time for $2 \times 10^{4} M_{\odot}$ clusters. Under this hypothesis it is possible that the most massive clusters of our sample (reaching typically $2 \times 10^{5} M_{\odot}$ ) can survive evaporation from internal processes for several gigayears. However, the disruption from tidal effects will be faster (Gieles et al. 2006).

Mass loss from stellar evolution (40\% to $60 \%$ of the mass over 12 Gyr; Kruijssen \& Lamers 2008; Sippel et al. 2012), from gas cloud encounters, and from tidal harassment still needs to be taken into account. The study of these two mass-loss processes goes beyond the scope of our paper. Given the very gaseous environment of these TDGs and the high gas turbulence, we may expect the latter two processes to be more efficient than in isolated and kinematically relaxed systems. At the same time, star clusters in such a gas-rich environment may continue to accrete gas from their surroundings (Pflamm-Altenburg \& Kroupa 2009; Naiman et al. 2011; Li et al. 2016).
Thus, if the YMCs of these TDGs are similar to YMCs observed in other environments in terms of density, we do not expect these dwarfs to form a system of massive star clusters that could last for a Hubble time. This conclusion is mitigated when we allow for a lower star density, which remains empirically unconstrained given our spatial resolution.

\subsection{Evolution of the TDGs}

The $\Lambda$ CDM paradigm predicts a different DM content for two classes of dwarf galaxies: TDGs, formed during interactions, which should be devoid of DM, and normal dwarf galaxies, such as the dwarf ellipticals (dE), dwarf irregulars, or BCDGs, formed inside a DM halo. This difference in DM would result in different kinematics and provide us with a new test for the $\Lambda$ CDM paradigm (see, e.g., Kroupa et al. 2010). Even though the absence of DM in TDGs is predicted from numerical simulations (Bournaud \& Duc 2006; Wetzstein et al. 2007; Bournaud et al. 2008), it is hard to prove because these are young and turbulent systems. Under the assumption of dynamical equilibrium, which is suggested by simulations to occur in less than one orbital time (Bournaud \& Duc 2006; Bournaud et al. 2007), HI kinematics are consistent with a purely baryonic content (Lelli et al. 2015). The kinematics of old TDGs need to be investigated. They are kinematically relaxed, and such an investigation might confirm such a purely baryonic content. If this were the case, it would meant that TDGs need to be distinguished from dEs.

Tidal dwarf galaxies are known to be outliers in the luminositymetallicity relation (e.g., Duc et al. 2000; Weilbacher et al. 2003). However, for old gas-poor TDGs, it might be still very challening to obtain the metallicity from the stellar population with current observing facilities. Moreover, as the metallicity of the host is a decreasing function of redshift, it might be argued that the deviation from the magnitude-metallicity relation will decrease, which will make it harder to separate old and same-age TDGs and dEs. Tidal dwarf galaxies are also known to be outliers in the size-mass relation for dwarf galaxies: they have unusually large effective radii for their mass (Duc et al. 2014).

A final means to distinguish these two categories could be to use their stellar cluster content (Dabringhausen \& Kroupa 2013). Dwarf ellipticals are known to host a significant number of GCs compared to their mass, with specific frequencies reaching up to 100 (Peng et al. 2008; Georgiev et al. 2010). Our analysis showed that even quite massive star clusters may form in TDGs. Some may be able to survive for several gigayears and thus be visible in rather old TDGs, but they will likely evaporate within a Hubble time because their SFR is too low to form clusters that are massive enough to survive evaporation for several gigayears. For a Hubble time, the minimum mass would be around the turnover value for the GC mass function (GCMF), $2 \times 10^{5} M_{\odot}$ (Fall \& Zhang 2001; Jordán et al. 2007).

Moreover, because a TDG potential well does not trap a significant amount of DM or old stars from the host, it might be argued that the capture of GCs from the host, which are kinematically coupled to either the DM halo or the bulge component (see review by Brodie \& Strader 2006), is also unlikely. This will be verified for our system in a future paper that will focus on the old cluster population, as described in Sect. 2. The accretion of old GCs onto TDGs also needs to be investigated by means of numerical simulations to understand the effect of varying the orbital parameters.

However, the conditions at higher redshift are most likely different because the host galaxy is likely to have a more substantial gas component (see, e.g., Combes et al. 2013). The TDG formation at high redshift has been studied very little, but simulations 
by Wetzstein et al. (2007) showed that more gas-rich disk galaxies are more likely to form TDGs, and Elmegreen et al. (2007b) found five young TDG candidates at $z=0.15-0.7$ that have higher stellar masses than typical local TDGs (up to $5 \times 10^{9} M_{\odot}$ ). As claimed by the latter, the higher velocity dispersion of both the gaseous and stellar components of higher redshift galaxies could lead to Jeans masses of up to $10^{10} M_{\odot}$ in tidal tails.

It might therefore be argued that at a given higher redshift, TDGs will have higher gas masses and higher SFRs. If star cluster formation at this cosmic epoch follows the empirical relation between the SFR of a galaxy and the magnitude of its brightest star cluster, given that the stellar models we used predict an $M_{V}=-12.4 \mathrm{mag}$ for a $10 \mathrm{Myr}$ old cluster of $2 \times 10^{5} M_{\odot}$, then an SFR of 5-10 $M_{\odot} \mathrm{yr}^{-1}$ would be sufficient to form some clusters more massive than the peak of the GCMF, which would be able to survive cluster dissolution for a Hubble time.

Although our analysis shows that TDGs formed under the current conditions are not likely to keep a GC system, more investigation is needed to understand whether TDGs that formed at higher redshifts would be able to harbor a GC system until the present epoch, and if they might be distinguished from other dwarfs using this criterion. Recently, two UDG candidates, DF2 and DF4, were found to share several of the properties expected for TDGs: a putative lack of DM, a large effective radius, and the proximity of a massive galaxy (van Dokkum et al. 2018a), which led to speculation of a tidal origin. The DM of this galaxy is still the subject of intense debate in the community (see, e.g., Martin et al. 2018; Trujillo et al. 2019; Blakeslee \& Cantiello 2018; Emsellem et al. 2019; Danieli et al. 2019). However, one unique feature is its large number of massive GCs (van Dokkum et al. 2018b). Fensch et al. (2019) found that the metallicity of the stellar body of DF2 and its GCs could be consistent with DF2 being an old TDG. However, a massive TDG like those around NGC 5291 did not form such massive clusters.

\subsection{Link to the formation of GCs in high-redshift galaxies}

A prevailing theory for the formation of the metal-rich population of GC around present-day massive galaxies is that they may have formed in the star-forming disk of the host galaxy at high redshift (Shapiro et al. 2010; Kruijssen 2012), when their morphology was dominated by 5 to $10 \mathrm{UV}$-bright giant clumps (mass $\sim 10^{7-9} M_{\odot}$, radius $\sim 1-3 \mathrm{kpc}$, Cowie et al. 1996; Elmegreen et al. 2009). A resolved study of clustered star formation in these clumps is unfortunately not possible with current instrumentation, except in some fortuitous cases of strong gravitational lensing (Cava et al. 2018). Thus local analogs are often used as laboratories to investigate the possible ISM and star cluster formation, such as the nearby BCDGs (Elmegreen et al. 2012b). In particular, they have been shown to be very efficient at forming YMCs (Östlin et al. 2003; Adamo et al. 2010; Lagos et al. 2011, and Sect. 4.4), although BCDGs are characterized by high gas fractions and turbulence (see, e.g., Lelli et al. 2014), similar to what is expected for higher redshift galaxies. However they usually have low to very low metallicities (typically $0.2 Z_{\odot}$, Zhao et al. 2013), while the giant clumps at high redshift already reach moderate metallicity, between one-third and half solar (Erb et al. 2006; Cresci et al. 2010; Zanella et al. 2015), which may have a strong effect on gas fragmentation (Krumholz \& Dekel 2012). Moreover, most of their stellar mass resides in an old stellar component (Loose et al. 1986; Papaderos et al. 1996), which means that these systems are dynamically old.

The TDGs are gas-rich, dynamically young, and have moderate metallicity. They are therefore probably better analogs to the clumps of high-redshift galaxies. The high to very high CFEs (up to $50 \%$ ) observed in the TDGs we presented in this study suggest that the physical conditions in high-redshift galaxies could be very favorable to the formation of star clusters. Moreover, if the empirical relation between the SFR of a galaxy and the magnitude of its brightest star cluster holds at these redshifts because giant clumps have SFRs of about $1-10 M_{\odot} \mathrm{yr}^{-1}$ (Guo et al. 2012), they might be expected to produce star clusters more massive $2 \times 10^{5} M_{\odot}$. This is the likely threshold mass that would allow them to survive dissolution over a Hubble time (see previous subsection).

The molecular surface gas density of TDGs is much lower than that of high-redshift galaxies; it is lower by two orders of magnitude (Lisenfeld et al. 2016). Their depletion timescale is also higher by a factor of 10 (2 Gyr for TDGs, Braine et al. 2001, $0.2 \mathrm{Gyr}$ for $z \simeq 2$ galaxies, Combes et al. 2013). Moreover, the tidal forces from the host are likely different, and are important for the formation of YMCs in colliding galaxies (Renaud et al. 2015) as well as for their survival (Baumgardt \& Makino 2003; Renaud et al. 2011). Numerical simulation work is therefore still needed to understand cluster formation in the giant clumps of high-redshift galaxies.

\section{Conclusion}

We investigated star cluster formation and evolution in three TGDs, whose physical properties differs from those of starbursting dwarfs. In particular, they are gas rich, highly turbulent, and their gas metallicity is already enriched to up to half solar.

The three TDGs are located in a huge collisional ring around NGC 5291. We observed this system with the HST using five broad bands from the near-UV to the near-IR. The photometry was extracted using PSF and Sérsic fitting, and we compared the obtained SED with stellar evolution models using the CIGALE code.

We find that star clusters are observed in TDGs, with masses of up to $10^{5} M_{\odot}$, with a mass distribution similar to those observed in other star cluster-forming systems. After taking into account the effect of the extinction-age degeneracies, we studied the star cluster formation efficiency in the TDGs. We showed that the three TDGs have high CFEs, above $30 \%$, with an average of $42 \%$. This is comparable to BCDGs, but with a lower SFR surface density, a higher metallicity and without being bona fide merging systems. The full sample of TDGs is located 2.5 to $3.8 \sigma$ above the relations from the literature. There may be uncertainties that are not yet recognized that still allow a constant CFE at this time (see, e.g., Chandar et al. 2017), and more data are needed for similar special galaxy types. Nevertheless, our results suggest that such a constant CFE relation would have a large scatter, and that there would be structure within this scatter that would be correlated with galaxy type and/or environment.

We next probed the existence of intermediate-age clusters, which could have formed during the early stages of the formation of the gaseous ring structure and may have survived for several 100 Myr. The fact that we could find some of them shows that cluster formation started early and we can expect the survival of young massive (above $10^{4} M_{\odot}$ ) star clusters from the formation of their host dwarf to several $100 \mathrm{Myr}$. However, if they have a similar density to what is observed for YMCs in other known environments (BCDGs, mergers), they might be present for a few gigayears but would be destroyed in a Hubble time because of relaxation-driven dissolution effects. If TDGs that formed at high redshift have a higher SFR, we may expect them to form more massive clusters that would be able to survive cluster dissolution for a Hubble time. 
Acknowledgements. MB acknowledges support of FONDECYT regular grant 1170618. Support for Program number HST-GO-14727 was provided by NASA through a grant from the Space Telescope Science Institute, which is operated by the Association of Universities for Research in Astronomy, Incorporated, under NASA contract NAS5-26555. DME acknowledges support from grant HST-GO14727.002-A. B.G.E. acknowledges support from grant HST-GO-14727.004-A. EB acknowledges support from the UK Science and Technology Facilities Council [grant number ST/M001008/1]. FR acknowledges support from the Knut and Alice Wallenberg Foundation.

\section{References}

Adamo, A., Zackrisson, E., Östlin, G., \& Hayes, M. 2010, ApJ, 725, 1620

Adamo, A., Östlin, G., \& Zackrisson, E. 2011, MNRAS, 417, 1904

Anders, P., Bissantz, N., Fritze-v. Alvensleben, U., \& de Grijs, R. 2004, MNRAS, 347, 196

Ashman, K. M., \& Zepf, S. E. 1992, ApJ, 384, 50

Bastian, N. 2008, MNRAS, 390, 759

Bastian, N., Adamo, A., Schirmer, M., et al. 2014, MNRAS, 444, 3829

Baumgardt, H., \& Makino, J. 2003, MNRAS, 340, 227

Bertin, E., \& Arnouts, S. 1996, A\&AS, 117, 393

Bigiel, F., Leroy, A., Walter, F., et al. 2008, AJ, 136, 2846

Bik, A., Östlin, G., Hayes, M., et al. 2015, A\&A, 576, L13

Billett, O. H., Hunter, D. A., \& Elmegreen, B. G. 2002, AJ, 123, 1454

Blakeslee, J. P., \& Cantiello, M. 2018, Res. Notes Am. Astron. Soc., 2, 146

Boquien, M., Duc, P.-A., Braine, J., et al. 2007, A\&A, 467, 93

Boquien, M., Duc, P.-A., Wu, Y., et al. 2009, AJ, 137, 4561

Boquien, M., Burgarella, D., Roehlly, Y., et al. 2019, A\&A, 622, A103

Bournaud, F., \& Duc, P.-A. 2006, A\&A, 456, 481

Bournaud, F., Duc, P.-A., Amram, P., Combes, F., \& Gach, J.-L. 2004, A\&A, 425,813

Bournaud, F., Duc, P.-A., Brinks, E., et al. 2007, Science, 316, 1166

Bournaud, F., Duc, P.-A., \& Emsellem, E. 2008, MNRAS, 389, L8

Boutloukos, S. G., \& Lamers, H. J. G. L. M. 2003, MNRAS, 338, 717

Bouwens, R. J., van Dokkum, P. G., Illingworth, G. D., et al. 2017, ArXiv e-prints [arXiv:1711.02090] unpublished

Braine, J., Duc, P.-A., Lisenfeld, U., et al. 2001, A\&A, 378, 51

Brodie, J. P., \& Strader, J. 2006, ARA\&A, 44, 193

Bruzual, G., \& Charlot, S. 2003, MNRAS, 344, 1000

Burgarella, D., Buat, V., \& Iglesias-Páramo, J. 2005, MNRAS, 360, 1413

Cava, A., Schaerer, D., Richard, J., et al. 2018, Nat. Astron., 2, 76

Chabrier, G. 2003, ApJ, 586, L133

Chandar, R., Fall, S. M., Whitmore, B. C., \& Mulia, A. J. 2017, ApJ, 849, 128

Combes, F., García-Burillo, S., Braine, J., et al. 2013, A\&A, 550, A41

Côté, P., Marzke, R. O., \& West, M. J. 1998, ApJ, 501, 554

Cowie, L. L., Songaila, A., Hu, E. M., \& Cohen, J. G. 1996, AJ, 112, 839

Cresci, G., Mannucci, F., Maiolino, R., et al. 2010, Nature, 467, 811

Dabringhausen, J., \& Kroupa, P. 2013, MNRAS, 429, 1858

Danieli, S., van Dokkum, P., Conroy, C., Abraham, R., \& Romanowsky, A. J. 2019, ApJ, 874, L12

de Grijs, R., Anders, P., Zackrisson, E., \& Östlin, G. 2013, MNRAS, 431, 2917

Duc, P.-A., \& Mirabel, I. F. 1998, A\&A, 333, 813

Duc, P. A., \& Mirabel, I. F. 1999, IAU Symp., 186, 61

Duc, P.-A., Brinks, E., Springel, V., et al. 2000, AJ, 120, 1238

Duc, P.-A., Paudel, S., McDermid, R. M., et al. 2014, MNRAS, 440, 1458

Elmegreen, B. G., \& Efremov, Y. N. 1997, ApJ, 480, 235

Elmegreen, D. M., \& Elmegreen, B. G. 2017, ApJ, 851, L44

Elmegreen, D. M., Elmegreen, B. G., Ferguson, T., \& Mullan, B. 2007a, ApJ, 663,734

Elmegreen, D. M., Elmegreen, B. G., Ravindranath, S., \& Coe, D. A. 2007b, ApJ, 658, 763

Elmegreen, B. G., Elmegreen, D. M., Fernandez, M. X., \& Lemonias, J. J. 2009 ApJ, 692, 12

Elmegreen, B. G., Malhotra, S., \& Rhoads, J. 2012a, ApJ, 757, 9

Elmegreen, B. G., Zhang, H.-X., \& Hunter, D. A. 2012b, ApJ, 747, 105

Emsellem, E., van der Burg, R. F. J., Fensch, J., et al. 2019, A\&A, 625, A76

Erb, D. K., Shapley, A. E., Pettini, M., et al. 2006, ApJ, 644, 813

Fall, S. M., \& Zhang, Q. 2001, ApJ, 561, 751

Fall, S. M., Chandar, R., \& Whitmore, B. C. 2005, ApJ, 631, L133

Fensch, J., Duc, P.-A., Weilbacher, P. M., Boquien, M., \& Zackrisson, E. 2016, A\&A, 585, A79

Fensch, J., Renaud, F., Bournaud, F., et al. 2017, MNRAS, 465, 1934

Fensch, J., van der Burg, R. F. J., Jerabkova, T., et al. 2019, A\&A, 625, A77

Forbes, D. A., Bastian, N., Gieles, M., et al. 2018, Proc. R. Soc. London Ser. A, 474, 20170616

Gaia Collaboration (Brown, A. G. A., et al.) 2018, A\&A, 616, A1

Georgiev, I. Y., Puzia, T. H., Goudfrooij, P., \& Hilker, M. 2010, MNRAS, 406, 1967
Gieles, M., Portegies Zwart, S. F., Baumgardt, H., et al. 2006, MNRAS, 371, 793

Giovannoli, E., Buat, V., Noll, S., Burgarella, D., \& Magnelli, B. 2011, A\&A, 525, A 150

Goddard, Q. E., Bastian, N., \& Kennicutt, R. C. 2010, MNRAS, 405, 857

Goodwin, S. P., \& Bastian, N. 2006, MNRAS, 373, 752

Gordon, K. D., Clayton, G. C., Misselt, K. A., Landolt, A. U., \& Wolff, M. J. 2003, ApJ, 594, 279

Guo, Y., Giavalisco, M., Ferguson, H. C., Cassata, P., \& Koekemoer, A. M. 2012, ApJ, 757, 120

Hénon, M. 1961, Annales d'Astrophysique, 24, 369

Hunter, D. A., Elmegreen, B. G., \& Gehret, E. 2016, AJ, 151, 136

Johnson, L. C., Seth, A. C., Dalcanton, J. J., et al. 2016, ApJ, 827, 33

Jordán, A., McLaughlin, D. E., Côté, P., et al. 2007, ApJS, 171, 101

Kennicutt, Jr., R. C., Hao, C.-N., Calzetti, D., et al. 2009, ApJ, 703, 1672

Kim, J.-H., Ma, X., Grudić, M. Y., et al. 2018, MNRAS, 474, 4232

Kimm, T., Cen, R., Rosdahl, J., \& Yi, S. K. 2016, ApJ, 823, 52

Knierman, K. A., Gallagher, S. C., Charlton, J. C., et al. 2003, AJ, 126, 1227

Koekemoer, A. M. 2002, HST Dither Handbook

Kroupa, P., \& Boily, C. M. 2002, MNRAS, 336, 1188

Kroupa, P., Famaey, B., de Boer, K. S., et al. 2010, A\&A, 523, A32

Kruijssen, J. M. D. 2012, MNRAS, 426, 3008

Kruijssen, J. M. D. 2015, MNRAS, 454, 1658

Kruijssen, J. M. D., \& Lamers, H. J. G. L. M. 2008, A\&A, 490, 151

Krumholz, M. R., \& Dekel, A. 2012, ApJ, 753, 16

Lagos, P., Telles, E., Nigoche-Netro, A., \& Carrasco, E. R. 2011, AJ, 142, 162

Larsen, S. S. 2002, AJ, 124, 1393

Larsen, S. S., \& Richtler, T. 2000, A\&A, 354, 836

Larsen, S. S., Brodie, J. P., \& Hunter, D. A. 2004, AJ, 128, 2295

Lelli, F., Verheijen, M., \& Fraternali, F. 2014, A\&A, 566, A71

Lelli, F., Duc, P.-A., Brinks, E., et al. 2015, A\&A, 584, A113

Li, H., \& Gnedin, O. Y. 2014, ApJ, 796, 10

Li, C., de Grijs, R., Deng, L., et al. 2016, Nature, 529, 502

Lisenfeld, U., Braine, J., Duc, P. A., et al. 2016, A\&A, 590, A92

Lofthouse, E. K., Kaviraj, S., Conselice, C. J., Mortlock, A., \& Hartley, W. 2017, MNRAS, 465, 2895

Loose, H. H., \& Thuan, T. X. 1986, in Star-forming Dwarf Galaxies and Related Objects, eds. D. Kunth, T. X. Thuan, J. Tran Thanh Van, J. Lequeux, \& J. Audouze, 73

Lotz, J. M., Miller, B. W., \& Ferguson, H. C. 2004, ApJ, 613, 262

Martin, N. F., Collins, M. L. M., Longeard, N., \& Tollerud, E. 2018, ApJ, 859, L5

Messa, M., Adamo, A., Calzetti, D., et al. 2018, MNRAS, 477, 1683

Mullan, B., Konstantopoulos, I. S., Kepley, A. A., et al. 2011, ApJ, 731, 93

Naiman, J. P., Ramirez-Ruiz, E., \& Lin, D. N. C. 2011, ApJ, 735, 25

Noll, S., Burgarella, D., Giovannoli, E., et al. 2009, A\&A, 507, 1793

Östlin, G., Zackrisson, E., Bergvall, N., \& Rönnback, J. 2003, A\&A, 408, 887

Papaderos, P., Loose, H.-H., Thuan, T. X., \& Fricke, K. J. 1996, A\&AS, 120, 207

Peebles, P. J. E. 1984, ApJ, 277, 470

Peng, C. Y., Ho, L. C., Impey, C. D., \& Rix, H.-W. 2002, AJ, 124, 266

Peng, E. W., Jordán, A., Côté, P., et al. 2008, ApJ, 681, 197

Peng, C. Y., Ho, L. C., Impey, C. D., \& Rix, H.-W. 2010, AJ, 139, 2097

Perret, V., Renaud, F., Epinat, B., et al. 2014, A\&A, 562, A1

Pflamm-Altenburg, J., \& Kroupa, P. 2009, MNRAS, 397, 488

Portegies Zwart, S. F., McMillan, S. L. W., \& Gieles, M. 2010, ARA\&A, 48, 431

Renaud, F. 2018, New A Rev., 81, 1

Renaud, F., Gieles, M., \& Boily, C. M. 2011, MNRAS, 418, 759

Renaud, F., Bournaud, F., \& Duc, P.-A. 2015, MNRAS, 446, 2038

Renaud, F., Agertz, O., \& Gieles, M. 2017, MNRAS, 465, 3622

Rodighiero, G., Daddi, E., Baronchelli, I., et al. 2011, ApJ, 739, L40

Salpeter, E. E. 1955, ApJ, 121, 161

Shapiro, K. L., Genzel, R., \& Förster Schreiber, N. M. 2010, MNRAS, 403, L36

Sippel, A. C., Hurley, J. R., Madrid, J. P., \& Harris, W. E. 2012, MNRAS, 427, 167

Tonini, C. 2013, ApJ, 762, 39

Trujillo, I., Beasley, M. A., Borlaff, A., et al. 2019, MNRAS, 486, 1192 van Dokkum, P. G. 2001, PASP, 113, 1420

van Dokkum, P., Danieli, S., Cohen, Y., et al. 2018a, Nature, 555, 629

van Dokkum, P., Cohen, Y., Danieli, S., et al. 2018b, ApJ, 856, L30

Vanzella, E., Castellano, M., Meneghetti, M., et al. 2017a, ApJ, 842, 47

Vanzella, E., Calura, F., Meneghetti, M., et al. 2017b, MNRAS, 467, 4304

Weilbacher, P. M., Duc, P. A., \& Fritze-v. Alvensleben, U. 2003, A\&A, 397, 545

Wetzstein, M., Naab, T., \& Burkert, A. 2007, MNRAS, 375, 805

Whitmore, B. C., Chandar, R., \& Fall, S. M. 2007, AJ, 133, 1067

Whitmore, B. C., Chandar, R., Kim, H., et al. 2011, ApJ, 729, 78

Zanella, A., Daddi, E., Le Floc'h, E., et al. 2015, Nature, 521, 54

Zhao, Y., Gao, Y., \& Gu, Q. 2013, ApJ, 764, 44 


\section{Appendix A: Single-band images for TDG SW and S}

The images corresponding to Fig. 1 for TDG S and SW are presented in Figs. A.1 and A.2.
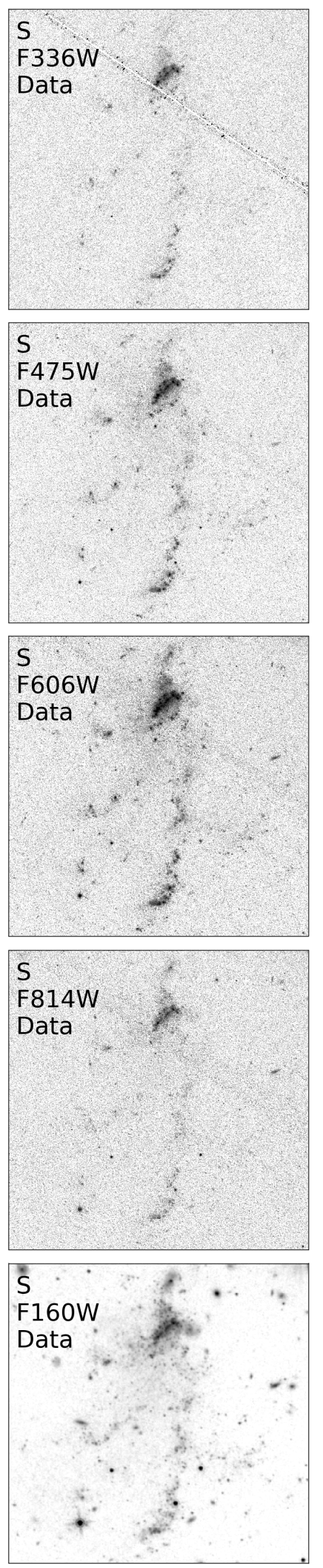

$\mathrm{S}$

F336W

Model

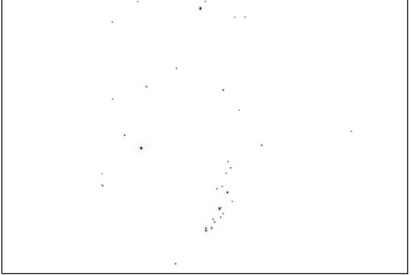

S

F475W

Model

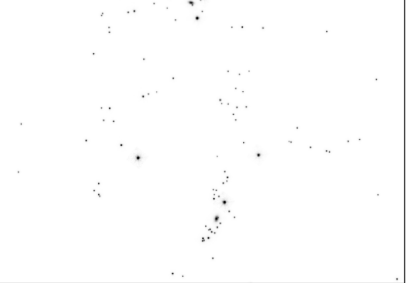

S

F606W

Model
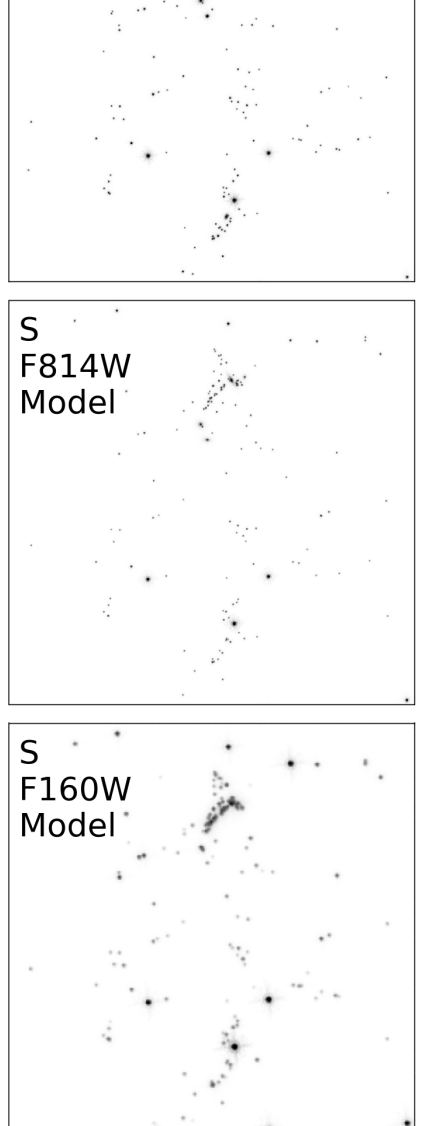
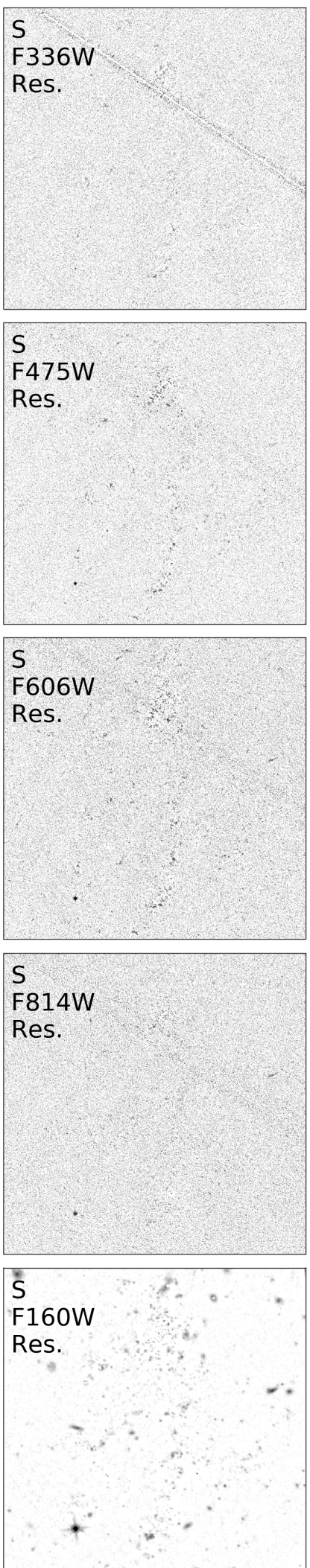

Fig. A.1. Data, model, and residual images for the TDG S. For each filter we show the data in the left column, the model in the middle column, and the residual in the right column. From top to bottom: F336W, F475W, F606W, F814W, and F160W. 

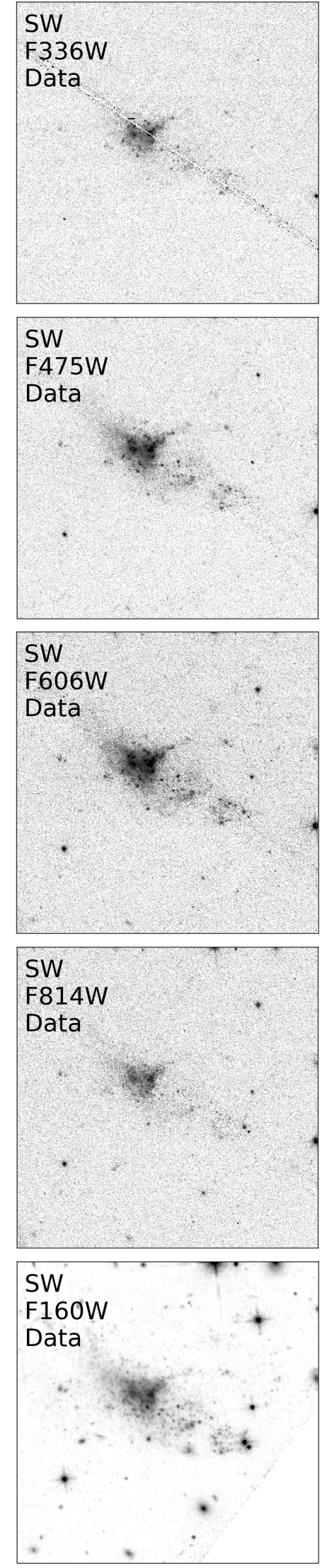
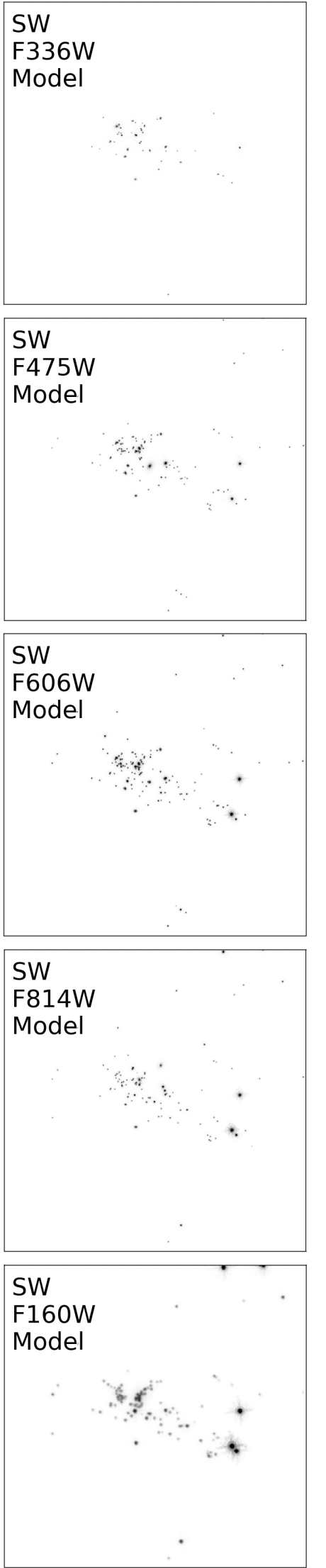
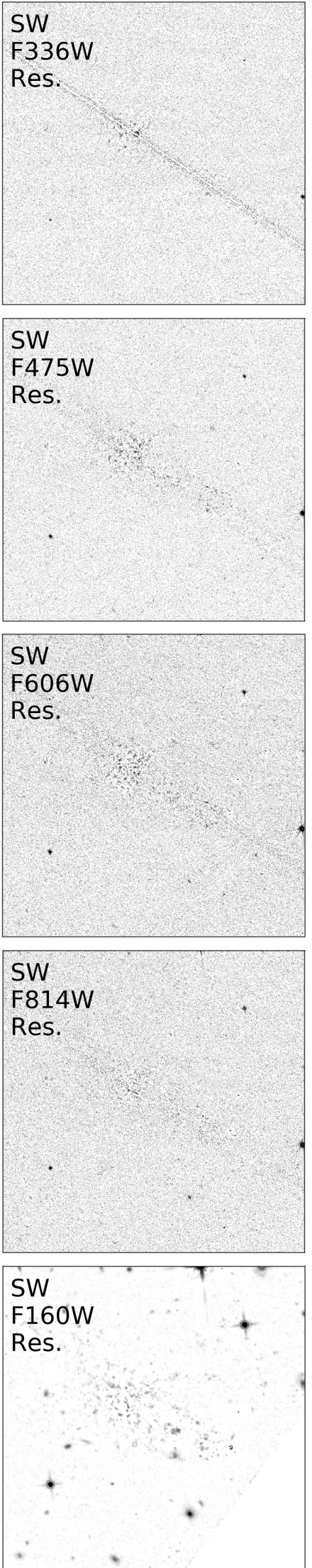

Fig. A.2. Data, model, and residual images for the TDG SW. For each filter we show the data in the left column, the model in the middle column, and the residual in the right column. From top to bottom: F336W, F475W, F606W, F814W, and F160W. 


\section{Appendix B: Sample selection}

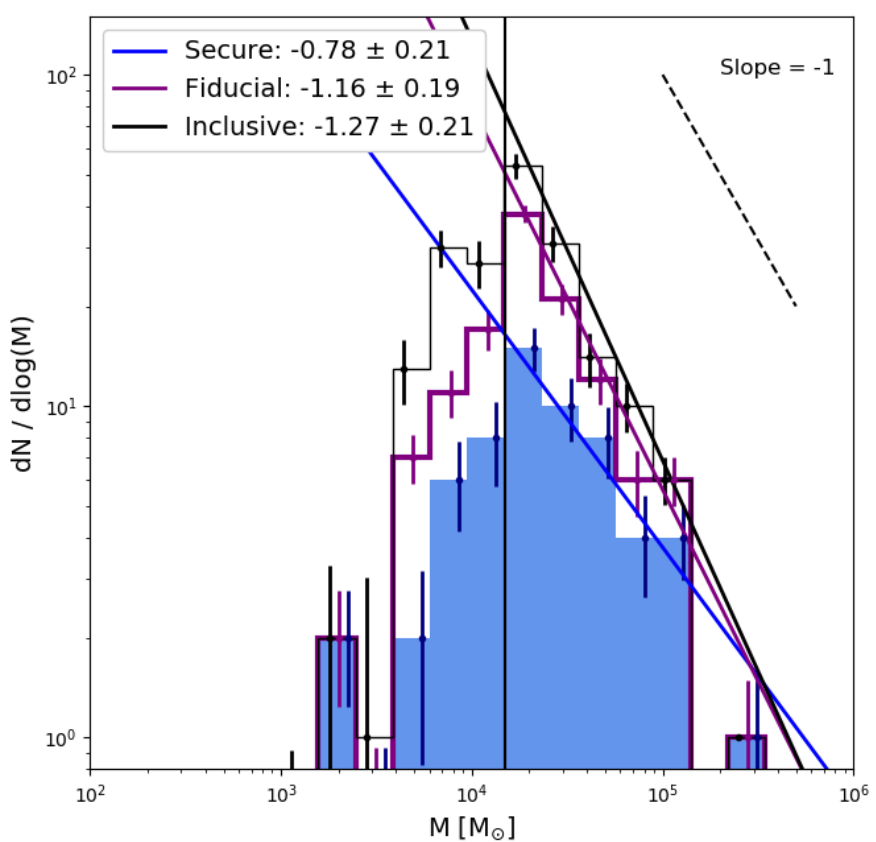

Fig. B.1. Same legend as for Fig. 6. The blue (black) histogram and line show the mass histogram and fit for the secure (inclusive) samples.

The degeneracy between age and extinction prevented us from building a complete sample. We thus used a sample that was defined using the age PDF output from CIGALE. In the following we consider two other samples.

- Secure sample: the retrieved age is younger than $30 \mathrm{Myr}$ and $P[$ age $<40]>0.9$.

- Fiducial sample: the mode of the age PDF is below $30 \mathrm{Myr}$ and $P$ age $<40]>0.5$. This sample was used in our main study.

- Inclusive sample: $P[0<$ age $<40]>0.1$, and we work with the mass obtained by using as new age prior [1 Myr, $30 \mathrm{Myr}$.

On the one hand, the secure sample only includes clusters that are securely younger than $30 \mathrm{Myr}$, but will miss all clusters affected by degeneracies and clusters with age PDFs that are not narrow enough. On the other hand, the inclusive sample includes most clusters that are younger than $30 \mathrm{Myr}$, but will include a significant number of older clusters that may resemble young clusters in our photometry. The secure sample is a subset of our fiducial study, which is a subset of the inclusive sample. These two samples may thus provide us with lower or upper limits.

The CMFs obtained for these three samples are shown in Fig. B.1. The secure and inclusive samples have a shallower and a steeper mass distribution, respectively.

The CFEs inferred from these two samples are summarized in Table B.1 and shown in Fig. B.2. The CFE for the secure (inclusive) sample can be considered as lower (upper) limits to the CFE of the genuine sample of clusters younger than $30 \mathrm{Myr}$. The offset between the data and the relations from the literature is given in Table B.2. When we consider the secure sample, the group defined by TDG N, SW, and $\mathrm{S}$ is $2.8 \sigma, 1.8 \sigma$, and $1.1 \sigma$ above the $\mathrm{K} 12, \mathrm{~J} 16$, and $\mathrm{C} 17$ relations, respectively. For the inclusive sample, the group defined by TDG N, SW, and S
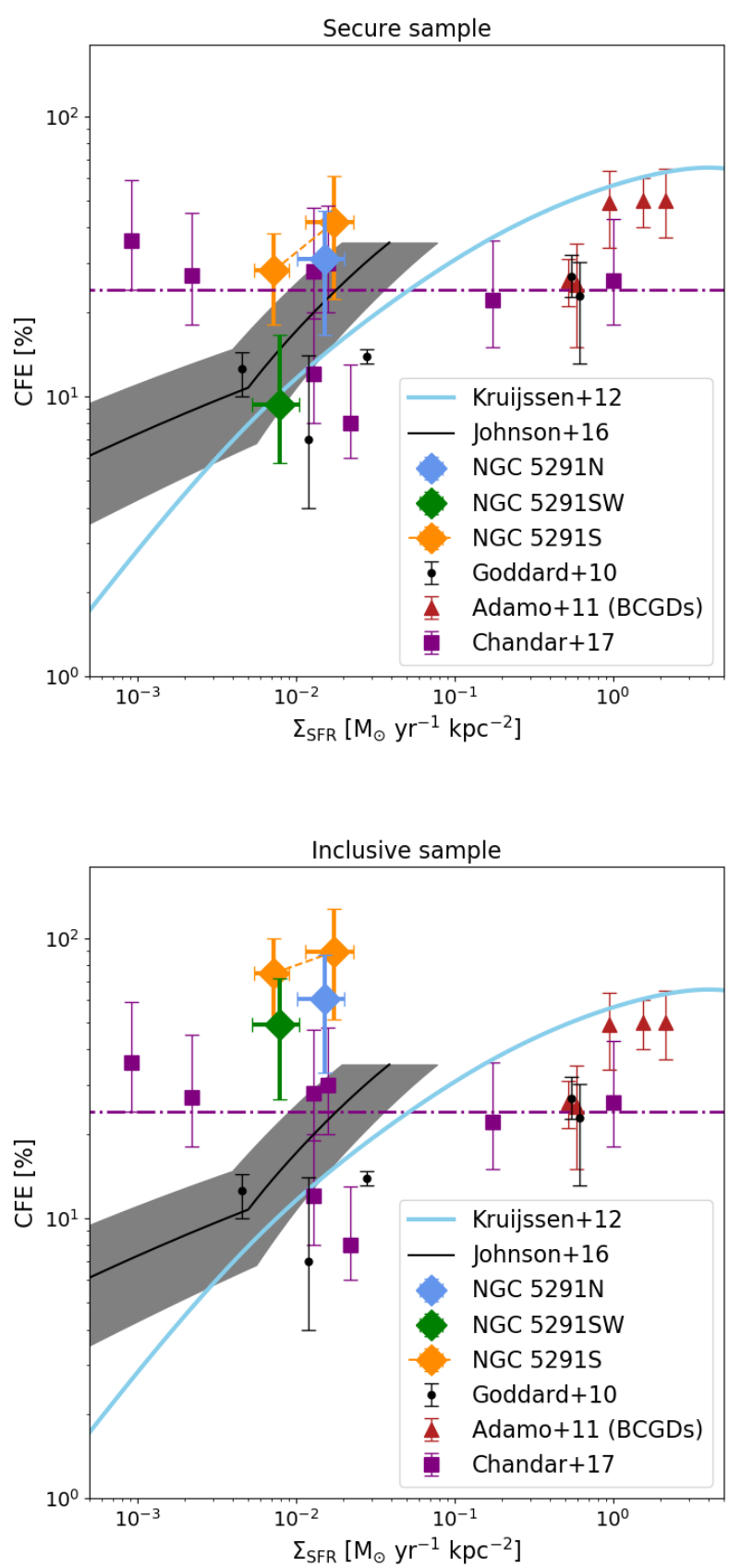

Fig. B.2. Same legend as for Fig. 8.

Table B.1. Values of the CFE for the three TDGs for the three age samples (see text).

\begin{tabular}{lcc}
\hline \hline & $\begin{array}{c}\text { CFE [\%] } \\
\text { Gecure }\end{array}$ & $\begin{array}{c}\text { CFE [\%] } \\
\text { Inclusive }\end{array}$ \\
\hline TDG N & $30_{-14}^{+15}$ & $60_{-27}^{+26}$ \\
TDG SW & $9_{-3}^{+7}$ & $48_{-22}^{+23}$ \\
TDG S & $28_{-10}^{+10}$ & $74_{-25}^{+25}$ \\
Including S* & $41_{-19}^{+18}$ & $89_{-38}^{+38}$ \\
\hline
\end{tabular}

is $4.3 \sigma, 3.7 \sigma$, and $3.3 \sigma$ above the $\mathrm{K} 12, \mathrm{~J} 16$, and $\mathrm{C} 17$ relations, respectively. 
A\&A 628, A60 (2019)

Table B.2. Significance, in standard deviation, of the offset between the TDGs and the relations from the literature.

\begin{tabular}{lccc}
\hline \hline Galaxy & K12 & $\mathrm{J} 16$ & $\mathrm{C} 17$ \\
\hline Secure sample & & & \\
TDG N & 1.1 & 0.4 & 0.3 \\
TDG SW & 0.0 & 0.0 & 0.0 \\
TDG S & 1.8 & 1.1 & 0.3 \\
Including S* & 1.4 & 0.9 & 0.8 \\
TDG N+SW+S & 2.8 & 1.8 & 1.1 \\
TDG N+SW+S* & 2.5 & 1.7 & 1.5 \\
\hline Inclusive sample & & & \\
TDG N & 1.8 & 1.4 & 1.3 \\
TDG SW & 1.6 & 1.4 & 1.0 \\
TDG S & 2.6 & 2.3 & 1.9 \\
Including S* & 2.0 & 1.7 & 1.7 \\
TDG N+SW+S & 4.3 & 3.7 & 3.3 \\
TDG N+SW+S* & 3.9 & 3.4 & 3.2 \\
\hline
\end{tabular}

